\title{
Evolution of a ring around the Pluto-Charon binary
}

\author{
Benjamin C. Bromley \\ Department of Physics \& Astronomy, University of Utah, \\ 115 S 1400 E, Rm 201, Salt Lake City, UT 84112 \\ bromley@physics.utah.edu \\ Scott J. Kenyon \\ Smithsonian Astrophysical Observatory, \\ 60 Garden St., Cambridge, MA 02138 \\ skenyon@cfa.harvard.edu
}

\begin{abstract}
We consider the formation of satellites around the Pluto-Charon binary. An early collision between the two partners likely produced the binary and a narrow ring of debris, out of which arose the moons Styx, Nix, Kerberos and Hydra. How the satellites emerged from the compact ring is uncertain. Here we show that a particle ring spreads from physical collisions and collective gravitational scattering, similar to migration. Around a binary, these processes take place in the reference frames of 'most circular' orbits, akin to circular ones in a Keplerian potential. Ring particles damp to these orbits and avoid destructive collisions. Damping and diffusion also help particles survive dynamical instabilities driven by resonances with the binary. In some situations, particles become trapped near resonances that sweep outward with the tidal evolution of the Pluto-Charon binary. With simple models and numerical experiments, we show how the PlutoCharon impact ring may have expanded into a broad disk, out of which grew the circumbinary moons. In some scenarios, the ring can spread well beyond the orbit of Hydra, the most distant moon, to form a handful of smaller satellites. If these small moons exist, New Horizons will find them.
\end{abstract}

Subject headings: Kuiper belt: general - planets and satellites: formation planets and satellites: rings - planet-disk interactions 


\section{Introduction}

With its rich system of satellites, the Pluto-Charon binary seems a miracle of planetary dynamics. Separated by about 17 Pluto radii $\left(1 R_{\mathrm{P}} \approx 1200 \mathrm{~km}\right.$; Young \& Binzel 1994; Young et al. 2007), Pluto and Charon have masses of $M_{\mathrm{P}} \approx 1.3 \times 10^{25} \mathrm{~g}$ and $M_{\mathrm{C}} \approx$ $1.5 \times 10^{24} \mathrm{~g}$ (Christy \& Harrington 1978; Buie et al. 2006). Their moons are not far away: Styx, Nix, Kerberos and Hydra are all packed between $37 R_{\mathrm{P}}$ and $60 R_{\mathrm{P}}$, on coplanar, nearly circular orbits with periods that are close to 3:4:5:6 resonances with the binary (Weaver et al. 2006; Showalter et al. 2011, 2012, 2013; Buie et al. 2013; Brozović et al. 2015; Showalter \& Hamilton 2015). The total mass of the satellites is a small fraction $(\lesssim 0.02 \%)$ of the binary's mass, roughly $3 \times 10^{19} \mathrm{~g}$ to $3 \times 10^{21} \mathrm{~g}$ (Buie et al. 2006; Brucker et al. 2009; Showalter et al. 2011, 2012; Youdin et al. 2012; Brozović et al. 2015). Resonances with the binary and the gravitational effects of the moons on each other keep the satellite system on the verge of chaotic disintegration (Tholen et al. 2008; Süli \& Zsigmond 2009; Winter et al. 2010; Peale et al. 2011; Youdin et al. 2012; Showalter \& Hamilton 2015). How the satellites arrived at their fragile orbits around Pluto and Charon is a mystery.

This delicate orbital architecture makes the Pluto-Charon system a challenge for theories of circumbinary planet formation (e.g., Moriwaki \& Nakagawa 2004; Quintana \& Lissauer 2006; Pierens \& Nelson 2007; Rafikov 2013; Bromley \& Kenyon 2015). The most promising scenario for the origin of the binary itself is that two dwarf planets had a grazing collision, leaving them intact but bound at a small separation of 4-5 $R_{\mathrm{P}}$ (McKinnon 1989; Stern 1992; Canup 2005, 2011). Pluto and Charon then tidally evolved, moving out to their present positions, locked synchronously together with a 6-day orbital period (Farinella et al. 1979; Dobrovolskis et al. 1997; Peale 1999; Cheng et al. 2014b). The impact likely produced a dynamically hot ring, confined to a region no more than a few times the binary's initial separation, inside the present-day orbit of Styx, the innermost satellite (Canup 2005, 2011).

In the impact scenario, the four moons come from the debris fragments (Stern et al. 2006; Canup 2005). After settling at orbital distances of 5-30 Pluto radii (Canup 2005), the debris circularizes into a narrow circumbinary ring. Over time the ring spreads radially outward, and small debris particles within it grow through coagulation. The result is a satellite system that has expanded outward by a factor of three or more in orbital distance (Kenyon \& Bromley 2014). A major uncertainty in this picture is how the ring - either with fully formed satellites or smaller debris - can spread to the moons' current positions. A compelling idea is that the moons formed quickly and migrated outward, trapped in resonances that expanded as the binary tidally evolved (Ward \& Canup 2006). However, this mechanism depends on details of the tidal evolution, and no single model can place all of the moons in their present orbits (Lithwick \& Wu 2008; Cheng et al. 2014a). 
Despite difficulties with the Ward \& Canup (2006) resonant migration scenario, it has an important implication. Even if satellites were placed by hand in their present orbits just after the giant impact, then at least some would be lost when unstable resonances swept outward along with the expanding binary (Lithwick \& Wu 2008; Cheng et al. 2014a,b). Collisional damping and diffusion might mitigate the effects of the resonances, but only if satellites evolve in partnership with a reservoir of smaller particles to damp them (Walsh \& Levison 2015). Therefore, the key to putting the moons into their current orbits is likely coordination between growth of the satellites, the depletion of the smaller debris, the spreading of the ring, and the tidal evolution of the binary. The primary motivation for this work is to understand this interplay.

Here, with the Pluto-Charon system in mind, we investigate circumbinary ring dynamics to see how a compact ring of growing particles evolves. Through analytic estimates and numerical calculations, we examine the role of particle viscosity (Cook \& Franklin 1964; Goldreich \& Tremaine 1978) and gravity (Lin \& Papaloizou 1979a; Ward 1997). We highlight the dependence of the spreading rate on the state of the ring particles, particularly their random velocities within the ring, and we consider the effects of in situ satellite growth (Kenyon \& Bromley 2014). In developing simple parameterized models, we offer pathways from an initially compact ring around a short-period binary to the more extended system of moons we see today. Some models predict the formation of more small moons at orbital distances beyond Hydra.

In this paper, we outline the main physical phenomena that are important to the formation of a circumbinary satellite system like Pluto-Charon's ( 2 ). Then we give a general introduction to ring dynamics (§3), including the effects of a central binary on the ring (§4). Turning our analysis to Pluto-Charon, we describe models of ring evolution (§5), and conclude with some predictions for New Horizons ( $\S 6)$, now rapidly approaching the Pluto-Charon system (Stern 2008).

\section{Overview and Context}

The formation of the Pluto-Charon satellite system is a challenging problem involving a range of physical phenomena. The grazing collision scenario for the formation of the binary yields only a narrow, compact ring that somehow spreads, either from interactions between ring particles or with the binary. The time scale for these processes is uncertain. In some circumstances, the ring spreads rapidly through gravitational stirring and physical collisions. In other situations, the ring spreads slowly as a result of collisional damping with only long-range, weak gravitational interactions to drive the expansion. In both cases, 
resonance excitations from the binary sweep through the system and potentially destabilize satellite orbits. One of our primary goals is to understand how the ring expands as the orbit of the binary evolves. Figure 1 illustrates the main issues, along with the overall layout of the Pluto-Charon system. The following list provides more detail about characteristics of the binary, the ring around it, and the physical processes that guide the emergence of the satellite system.

- The circumbinary environment: the inner cavity. The Pluto-Charon binary provides a time-varying potential that complicates the nature of orbiting satellites (Lee \& Peale 2006). Circumbinary orbits near Pluto and Charon are largely unstable, leaving an inner cavity that is about twice the binary separation, just inside the orbit of Styx (e.g., the light gray shaded region in Figure 1, see also Holman \& Wiegert 1999; Musielak et al. 2005; Pichardo et al. 2005; Doolin \& Blundell 2011). Carved out by instabilities at overlapping resonances (Wisdom 1980) and by resonant-driven torque exchange (e.g., Goldreich \& Tremaine 1980; Mever-Vernet \& Sicardy 1987; Ward 1997), this cavity sets the inner boundary of the circumbinary ring. Due to uncertainties in this complex environment, we consider two approaches to the boundary: (i) loss of ring material by ejection and (ii) a balance between viscous inflow and the outward torque from Charon.

- Resonances. Aside from defining the edge of the inner cavity, unstable resonances lie within the inner cavity and (for eccentric binaries) at isolated larger orbital distances (e.g., Ward \& Canup 2006; Cheng et al. 2014b). Current analyses favor a circular Pluto-Charon orbit; resonances beyond the cavity's edge at the 3:1 commensurability are stable (e.g., Figure 3 , below). Initially, the binary probably had significant eccentricity (Canup 2005); resonances at higher order commensurabilities, include those at 4:1, 5:1, and 6:1, were then unstable. Here we explore the effect of these resonances in the context of ring dynamics (\$4.1) and show that sufficiently strong collisional damping stabilizes particles within these resonances.

- Stable circumbinary orbits. Despite the resonances, the binary has little impact on much of the orbital domain outside the inner cavity. As demonstrated by analytic theory (Lee \& Peale 2006; Leung \& Lee 2013) and numerical experiment (Bromley \& Kenyon 2015), non-resonant orbits experience no secular excitation or long-term torque exchange with the binary (\$4). Particles tend to follow trajectories that ebb and flow with the orbital motion of the binary. When swarms of particles experience orbital damping, they settle on a family of "most circular" paths that do not intersect, just like circular paths around a single central mass (Youdin \& Kenyon 2013). For a reference frame tied to a most circular orbit, the local dynamics within a ring around a 
binary is very similar to dynamics around a single central mass. After describing these features (\$4), we take advantage of them when modeling ring evolution (\$5)).

Ring stability considerations: total mass and particle size. We also consider the gravitational stability of a ring with velocity dispersion $v$, orbital frequency $\Omega$, and surface density $\Sigma$ orbiting Pluto-Charon. When ring particles achieve a balance between gravitational stirring and collisional damping, the velocity dispersion is roughly the escape velocity of the largest particles in the ring. Defining $G$ as the gravitational constant, the Toomre stability criterion, $v \Omega>\pi G \Sigma$, establishes stable configurations of ring parameters (e.g., Chiang \& Youdin 2010). Adopting a ring radius of $20 R_{\mathrm{P}}$, width of $5 R_{\mathrm{P}}$ and mass, $3 \times 10^{20} \mathrm{~g}$, comparable to the mass of the known satellites, the ring is stable if the velocity dispersion exceeds the escape velocity of a 10-meter icy particle, roughly $1 \mathrm{~cm} / \mathrm{s}$. Unless mergers produce larger particles which gravitationally stir the smaller particles up to their escape velocity, a ring composed of smaller particles is unstable. In most of the models discussed here, we assume a sea of particles with radii of $1 \mathrm{~km}$; rings composed of these particles are stable up to masses of $4 \times 10^{22} \mathrm{~g}$, roughly two orders of magnitude more massive than the binary's satellites.

Ring dynamics. We envision the spreading of the ring from its early compact configuration through particle interactions. When particle velocities exceed their escape speed, either from stirring or because they are in the Roche zone of the central mass, physical collisions dominate the dynamics (Cook \& Franklin 1964; Goldreich \& Tremaine 1978). Then, an effective collision-driven viscosity drives the evolution of the ring. Outside the Roche zone (which is only about $3 R_{\mathrm{P}}$ for Pluto) collisional damping reduces the relative particle speeds until gravitational interactions are important (Hornung et al. 1985; Shu \& Stewart 1985). Here, we provide a theoretical prescription for how these longer range interactions cause the ring to evolve. As illustrated in Figure 1, collisionally driven viscosity, with stirring by larger embedded particles, leads to fast migration. Purely gravitational spreading is comparatively slow.

- Evolution of the binary. Tidal evolution of the binary lies at the heart of all these issues. After the grazing impact, the binary has a tighter, more eccentric orbit. Tides circularize and expand the system into the current circular orbit (e.g., Cheng et al. 2014a). Our analysis considers how tidal evolution drives a set of sweeping mean motion resonances (e.g., Figure 1) which lead to the dynamical ejection of satellites (Cheng et al. 2014b). By including collisional damping and ring viscosity, we begin to show how growing satellites or their precursors experience resonant trapping, allowing them to migrate outward with the binary's tidal expansion. Alternatively, if the ring spreads quickly, we consider how satellites might survive, or at least reform, after the resonances sweep past. 
While the circumbinary nature of Pluto-Charon satellites make them unique in the Solar System, key physical processes governing their formation are also important to other Solar System objects. For example, the regular satellites of Jupiter grow within a viscously spreading circumprimary disk (see Lunine \& Stevenson 1982; Canup \& Ward 2002; Mosqueira \& Estrada 2003a,b; Mosqueira et al. 2010; Sasaki et al. 2010; Ward \& Canup 2010; Ogihara \& Ida 2012). Although gas dynamics is crucial to this evolution, our analysis addresses aspects of the spreading of large particles uncoupled from the gas. With its gas free environment, the Pluto-Charon satellite system is more akin to Saturn's rings (see Canup \& Esposito 1995; Porco et al. 2007; Charnoz et al. 2010). Despite clear differences in the location of small particles relative to the Roche limit (e.g., mostly inside for Saturn and entirely outside for Pluto-Charon), both systems are driven by pure particle dynamics. To extend the theory of Saturn's rings to a ring of solids orbiting Pluto-Charon, we focus next on the physics of particle rings.

\section{Ring dynamics}

Planetary rings provide an excellent theoretical proving ground for planet formation. Saturn's rings famously became a subject for James Clerk Maxwell, and inspired a powerful theory for evolution within the Roche limit of the planet (Cook \& Franklin 1964; Goldreich \& Tremaine 1978). Inside this radial distance from Saturn, tidal forces inhibit particle growth. Thus, the theory concentrates on the radial spreading and velocity evolution of small, indestructible particles with negligible gravitational interactions. Beyond the Roche limit, rapid growth into small moons is a likely outcome; radial spreading and gravitational scattering within the rings remain secondary (Charnoz et al. 2010). For a ring of particles orbiting Pluto-Charon outside the Roche limit, gravitational interactions between large particles add to the spreading from small particles. Furthermore, dynamical spreading and particle growth occur simultaneously. To understand the evolution of this material, we require a more general treatment.

Here we establish a theory for describing the major influences on the evolution of a planetary ring. Following Goldreich \& Tremaine (1978), we discuss the role of physical collisions under the assumption that the particles rebound off one another as they collide, changing their trajectories but not their masses or radii (\$3.2). Inelastic collisions allow damping of random speeds (e.g., Bridges et al. 1984) and convert orbital energy into random motions, yielding an effective viscosity that spreads material radially (Jeffrevs 1947; Cook \& Franklin 1964; Goldreich \& Tremaine 1978, §3.3, below). We also consider the role of mutual gravitational interactions. Gravitational scattering affects the equilibrium veloc- 
ities that enter in the viscosity (Hornung et al. 1985; Shu \& Stewart 1985; Goldreich et al. 2004), as well as the radial spreading of particles from collective interactions similar to migration (Lin \& Papaloizou 1979a; Goldreich \& Tremaine 1980; Ward 1997). Finally, we give a prescription for the evolution of a ring in which particles grow. By incorporating all of these phenomena together, we construct simple models for ring evolution and lay the foundation for including this physics into full evolution codes (e.g., Weidenschilling et al. 1997; Kenyon \& Luu 1998; Kenyon \& Bromley 2014).

\subsection{Background}

Disks or rings around a central mass are a natural outcome in many astrophysical systems. Conservation of angular momentum, along with dissipative processes (e.g., radiative cooling of gas or inelastic collisions between particles) drive material toward these flattened configurations (e.g., Lynden-Bell \& Pringle 1974; Brahid 1976; Pringle 1981). In this section, we examine a ring of particles around a central point pass in the limit where the self-gravity of the ring is not important. We begin by establishing some basic physical scales and relationships between physical properties of the ring.

To model the ring, we first assume that it is made of identical particles with fixed radius $r$, mass $m$, and density $\rho$. A particle at an orbital distance $a$ has an orbital period

$$
T_{\mathrm{K}}=2 \pi \sqrt{a^{3} / G M}
$$

and Keplerian velocity

$$
v_{\mathrm{K}}=\sqrt{G M / a}
$$

where $M$ is the central mass and $G$ is the gravitational constant. We assume that the orbits of the ring particles are approximately circular and have instantaneous speeds close to $v_{\mathrm{K}}$.

For orbits near the central mass, tidal forces are strong. The Roche limit gives a critical value,

$$
a_{\mathrm{R}} \approx 1.5\left(\frac{M}{\rho}\right)^{1 / 3},
$$

inside of which massive particles are unable to hold themselves together by their bulk strength and self-gravity (Holsapple \& Michel 2006, 2008).

When particles are well outside of the Roche limit, they interact gravitationally with

other neighboring satellites (Salmon et al. 2010; Charnoz et al. 2011; Rosenblatt \& Charnoz 
2012). The gravitational range is characterized by the Hill radius,

$$
r_{\mathrm{H}}=a\left(\frac{m}{3 M}\right)^{1 / 3}
$$

The particle's Hill velocity,

$$
v_{\mathrm{H}}=a \sqrt{G m / r_{\mathrm{H}}},
$$

is the speed of a low-mass neighbor orbiting the particle at the Hill radius. Neighbors passing by at much faster speeds are undisturbed; slower objects are stirred to at least this speed (see Goldreich et al. 2004).

The fastest speed to which a particle can stir a less massive neighbor is approximately the escape speed at its surface (see Goldreich et al. 2004):

$$
v_{\mathrm{esc}}=\sqrt{2 G m / r}=\sqrt{8 \pi G \rho r^{2} / 3} \text {. }
$$

During an encounter, if the relative speed of an object and the satellite is small compared to $v_{\text {esc }}$, gravity influences the outcome. When the relative speed exceeds $v_{\text {esc }}$, gravitational interactions are much less important than physical collisions.

In a swarm of identical particles, the escape velocity provides an estimate of the characteristic speed of particles, $v$, as measured in a local Keplerian frame that follows a circular orbit in the ring midplane. The actual value of $v$, which we associate with "random motion" in this reference frame, depends on collision outcomes and physical conditions in the disk, such as the number density of particles $n(a)$, the surface (mass) density $\Sigma(a)$, and the vertical scale height $h(a)$. These properties of the disk are inter-related,

$$
\begin{aligned}
& n(a) \sim \frac{\Sigma}{2 h m} \approx \frac{2 \pi \Sigma}{m v T_{\mathrm{K}}} \\
& h(a) \sim \frac{v T_{\mathrm{K}}}{4 \pi},
\end{aligned}
$$

where we assume a vertical speed $v_{\mathrm{z}} \approx v / 2$ (e.g., Ohtsuki 1992, and references therein). Thus, the microscopic particle velocities and the broader structure of the disk are entwined throughout its evolution.

\subsection{Particle collisions}

Physical collisions between particles hold the key to the evolution of systems of satellites, moons, and planets. Collisions not only result in fragmentation or mergers; they also 
damp random particle motions and reduce collision speeds. In the balance lies the difference between growth or destruction of satellites in a ring. When particles collide, their material properties determine the outcome. For icy bodies, shattering or fragmentation occurs at speeds of $v \gtrsim 10^{3} \mathrm{~cm} / \mathrm{s}$ (Kenyon \& Bromley 2004, and references therein). At slower speeds, bouncing and sticking can occur. Because high-speed collisions produce smaller bodies that can dynamically cool larger ones, collisions are essential to mergers and growth (e.g., Youdin \& Kenyon 2013).

A first step toward assessing the role of collisions is to estimate $T_{\text {col }}$, the characteristic time between collisions for a single particle. If particle velocities are comparable to or larger than their escape velocities, simple kinetic theory (" $n v \sigma$ ") gives (e.g., Safronov 1969; Lissauer 1987; Wetherill \& Stewart 1993; Goldreich et al. 2004)

$$
T_{\mathrm{col}} \approx \frac{1}{n \sqrt{2} v \pi 4 r^{2}} \approx \frac{\rho r}{2 \sqrt{2} \pi \Sigma} T_{\mathrm{K}}
$$

where the cross-sectional area of $\pi(2 r)^{2}$ is appropriate to hard spheres, and the pairwise velocity $\sqrt{2} v$ is eliminated in the rightmost expression because of its relationship to the scale height, $h$ (Equation (8)); see Ohtsuki 1992). When the relative speeds fall below the typical escape speed, gravitational focusing increases the effective cross-section and reduces the collision time (Wetherill \& Cox 1985; Wetherill \& Stewart 1989; Spaute et al. 1991; Goldreich et al. 2004; Youdin \& Kenvon 2013, see \$3.4.2, below).

If collisions are inelastic, relative particle velocities are damped. Absent other effects, we can identify a damping time scale in terms of the evolution of random kinetic energy:

$$
\frac{1}{v^{2}} \frac{d v^{2}}{d t} \sim-\frac{1}{T_{\text {damp }}} \text {. }
$$

Thus, the damping time is

$$
T_{\text {damp }} \approx \frac{T_{\text {col }}}{\bar{f}_{\text {loss }}}
$$

where $\bar{f}_{\text {loss }}$ is the average fractional loss in kinetic energy per collision. Since the random speed $v$ is measured in the local Keplerian frame, the damping time characterizes how collisions circularize the orbits of ring particles and flatten the ring.

The parameter $\bar{f}_{\text {loss }}$ carries the details of collisions between ring particles. Following Porco et al. (2008), we adopt simple parameterizations of the coefficients of restitution, which set the ratio of the particle speeds before and after a collision. Treating the normal (N) and transverse $(\mathrm{T})$ directions separately, these coefficients are

$$
\begin{aligned}
\epsilon_{N} & =\min \left[\left(v_{N} / v_{*}\right)^{b}, 1\right] \\
\epsilon_{T} & =0.9
\end{aligned}
$$


where $v_{N}$ is the normal component of the relative velocity. Experiments suggest $v_{*}=$ $0.01 \mathrm{~cm} / \mathrm{s}$ and $b=-0.14$ for spheres of water ice (Supulver et al. 1995; Bridges et al. 1984; Porco et al. 2008). Averaged over all impact parameters, the loss parameter for a given collision speed $u$ is

$$
f_{\text {loss }}(u)=1-\left(\epsilon_{N}^{2}+\epsilon_{T}^{2}\right) / 2,
$$

if collisions are random as in a 3D ideal gas. Averaging over the distribution of relative speeds yields $\bar{f}_{\text {loss }}$ (Equation (11)). In a simple model with a Maxwell-Boltzmann distribution of relative speeds,

$$
\begin{aligned}
\bar{f}_{\text {loss }} & \approx \frac{\int_{0}^{\infty} u^{4} d u f_{\text {loss }}(u) \exp \left(-3 u^{2} / 4 v^{2}\right)}{\int_{0}^{\infty} u^{4} d u \exp \left(-3 u^{2} / 4 v^{2}\right)} \\
& \approx 0.60-0.44\left(\frac{v}{v_{*}}\right)^{-0.28} \quad\left[b=-0.14, v \gg v_{*}\right]
\end{aligned}
$$

In this case, the energy loss for $v \gg v_{*}$ is limited by grazing collisions and the value of $\epsilon_{T}$. Unfortunately, this value is not well constrained by experiment.

\section{3. $\quad$ Ring viscosity}

While physical collisions sap random kinetic energy from the ring, they also add to it. By redirecting their trajectories after rebounding, collisions convert orbital motion into random velocities (Goldreich \& Tremaine 1978). Thus, the eccentricities and inclinations of ring particles grow; the entire ring broadens in semimajor axis. The diffusive spreading of ring particles can be expressed formally in terms of a viscosity,

$$
\nu_{\mathrm{rad}} \sim 0.46 \frac{v^{2} T_{\mathrm{K}}}{2 \pi} \frac{\tau}{1+\tau^{2}},
$$

where the optical depth of particles in the ring is

$$
\tau \sim \frac{\pi r^{2} \Sigma}{m} \sim \frac{3 \Sigma}{4 \rho r}
$$

(Cook \& Franklin 1964; Goldreich \& Tremaine 1978, 1982). To illustrate how the viscosity $\nu_{\text {rad }}$ is associated with diffusive spreading of the ring, we consider a particle as it rebounds during a collision. If we are oblivious of the details, the collision outcome appears stochastic, yielding a random step with a radial component that is directed either inward or outward with equal probability. The magnitude of this step is roughly the particle's epicyclic radius,

$$
|\delta a| \sim a \frac{v}{v_{\mathrm{K}}} .
$$


Viewing this outcome as part of a random walk, with steps taken at the collision rate of $1 / T_{\text {col }}$ (Equation (9)), we can infer an effective diffusion coefficient,

$$
\nu_{\mathrm{rad}} \sim \frac{|\delta a|^{2}}{T_{\mathrm{col}}}=\frac{a^{2} v^{2}}{v_{\mathrm{K}}^{2} T_{\mathrm{col}}}=\frac{a^{2} v^{2}}{\left(2 \pi a / T_{\mathrm{K}}\right)^{2} \cdot\left(\rho r T_{\mathrm{K}} / \Sigma\right)}=\frac{v^{2} T_{\mathrm{K}} \Sigma}{4 \pi \rho r}=\frac{v^{2} T_{\mathrm{K}} \tau}{3 \pi},
$$

where the rightmost expression is the low optical depth limit of Equation (17) to within a factor of $\sim 2$. After $n$ steps (collisions), the typical radial displacement grows to

$$
\left|\delta a^{(n)}\right| \sim \sqrt{\nu t} \sim a \frac{v}{v_{\mathrm{K}}} \sqrt{t / T_{\mathrm{col}}}
$$

with time $t$ set to $n T_{\text {col }}$. We can take advantage of this formula in simulating particle diffusion. Using a random number generator we can send particles on random walks in orbital distance to mimic the behavior of a viscous ring (Bromley \& Kenyon 2013, also \$4.1, below).

Over many collisions involving an ensemble of particles, the ring broadens by some radial distance $|\Delta a|$. The time scale for this spreading is

$$
T_{\text {spread }} \sim \frac{\left|\Delta a^{2}\right|}{\nu_{\text {rad }}}
$$

This relationship explains the slow spreading of Saturn's rings. Doubling the annular width of the A ring requires $\sim 1$ Gyr (Charnoz et al. 2009). Slow spreading is a major challenge for models in which an impact ring around the Pluto-Charon binary expands in time to form the moons at their present location (Kenyon \& Bromley 2014). We return to this issue in $\$ 5$.

Because collisions move particles to regions with different Keplerian velocity, they tend to pump up the relative speed of the particles. If collisions are completely elastic, the relative velocities grow unchecked (Goldreich \& Tremaine 1978). For example, a particle in a sea of objects on circular orbits might scatter slightly inward or outward after a collision, so that it has a new speed relative to its new neighbors, $|\Delta v| \sim e|\Delta a|$. Subsequent collisions cause that particle to walk randomly in velocity, as well as in radial distance. By the $n^{\text {th }}$ interaction the typical speed has grown to

$$
\left|\Delta v^{(n)}\right|^{2} \sim c\left|\Delta v^{(n-1)}\right|^{2} \sim c^{n-1}|\Delta v|^{2}
$$

where $c$ is a constant greater than unity that depends on the details of the interactions (e.g., particle sizes, orbital distances). Formally, random speeds quickly diverge (e.g., Goldreich \& Tremaine 1978). 
In reality, collisions involve some loss of kinetic energy, providing a means to balance this process. Coefficients of restitution that decrease with speed - and therefore cause more kinetic energy loss at higher speeds - allow a ring to reach an equilibrium. If the collisional damping is weak, rings equilibrate at high speeds; highly inelastic collisions lead to small random motions and nearly circular orbits. The details of this balance between dynamical heating and collisional damping are buried in the magnitude of the equilibrium velocity, $v$ in Equation (17).

\subsection{Gravitational scattering}

In addition to physical collisions, pairwise gravitational encounters can also cause random deflections and thus contribute to ring viscosity (Hornung et al. 1985; Barge \& Pellat 1990; Ohtsuki 1999; Ohtsuki et al. 2002). From Equation (20), we can surmise how gravitational interactions affect $\nu_{\text {rad }}$. Gravitational stirring and dynamical friction (Weidenschilling 1989; Kenyon \& Luu 1998; Stewart \& Ida 2000; Kenyon \& Bromley 2001) drive the local velocity evolution; gravitational focusing makes the effective cross-section larger and reduces the collision time. Thus, stirring and focusing tend to increase the viscosity and drive the spreading of the ring. However, for rings in a steady state, stirring pumps up velocities until they exceed the escape speed of particles. Then, gravitational focusing becomes unimportant (see below) as collisions take over to regulate the velocities. Therefore, gravity's main role in the ring viscosity is to help to maintain the equilibrium velocity established by collisions. After all, only three things matter to the spreading of the ring: how many particles are in the ring $(\Sigma)$, how big they are $(r)$ and how fast they are moving relative to each other $(v)$.

Here we review some general aspects of gravitational interactions between ring particles. For particles outside the Roche limit, separated by distances of a few Hill radii, we describe pairwise interactions as Rutherford scattering (Lin \& Papaloizou 1979b). Working in a pair's

center-of-mass frame, we relate impact parameters to scattering angles. Translating these scattering outcomes to changes in orbital elements, we then derive how gravity between ring particles affects the overall evolution of the ring.

\subsubsection{Rutherford scattering}

Pairwise gravitational interactions between massive particles are described by Rutherford scattering. Following the traditional analysis (e.g., Lin \& Papaloizou 1979b) we consider two point particles with masses $m_{1}$ and $m_{2}$ on approaching trajectories in their center-of- 
mass frame with impact parameter $b$ and closing speed $v$. Using the total and reduced masses

$$
m=m_{1}+m_{2} \quad \text { and } \quad \delta m=\frac{m_{1} m_{2}}{m_{1}+m_{2}},
$$

we write equations of motion for the pair as a one-body problem in terms of the pair's relative position and velocity. We are typically interested only in the outcome of these encounters, described in terms of

$$
\Theta=2 \arctan \frac{G m}{v^{2} b},
$$

which is the angle between the initial and final relative velocities. Another quantity that is important to scattering outcomes is the distance of closest approach, $r_{\text {close }}$, derived from

$$
r_{\text {close }}^{2}=b^{2}-\frac{2 G m r_{\text {close }}}{v^{2}}=b^{2}-\frac{v_{\text {esc }}^{2}}{v^{2}}\left(r_{\text {close }} \cdot r\right),
$$

where $v_{\text {esc }}$ is the escape velocity of an individual particle of radius $r$. Between the distance of closest approach and the scattering angle, we distinguish between different outcomes: physical collisions ( $r_{\text {close }}$ is less than the sum of particle radii) are one possibility as described above. For purely gravitational encounters, we also have strong/random scattering $(\Theta \sim 1$, $\left.r_{\text {close }} \ll b\right)$ and weak scattering $\left(\Theta \ll 1, r_{\text {close }} \approx b\right)$. We consider these regimes next, starting with strong interactions.

\subsubsection{Gravitational focusing and random scattering}

When gravitational encounters are close and deflections are strong, physical collision rates can be boosted and relative velocities can be randomized. In a pairwise encounter, if the closing speed $v$ is small compared to the escape velocity of the largest particle in a pair, then the distance of closest approach $r_{\text {close }}$ is significantly smaller than the impact parameter $b$ (Equation (26). This effect is gravitational focusing. If we compare the cross section for physical collisions between identical particles to $b_{c}$, the maximum impact parameter that leads to a collision, then we get the focusing factor,

$$
f_{\text {foc }} \equiv \frac{\pi b_{c}^{2}}{\pi(2 r)^{2}}=1+\frac{1}{2} \frac{v^{2}}{v_{\mathrm{esc}}^{2}} .
$$

This factor quantifies the boost in the likelihood of physical collisions and in the collision rate (in the context of planet formation, see Greenzweig \& Lissauer 1990; Spaute et al. 1991; Wetherill \& Stewart 1993). The overall rate of ring spreading is increased, with the viscosity parameter $\nu_{\text {rad }}$ augmented by a factor $f_{\text {foc }}$. 
Even in the absence of collisions, strong gravitational encounters can affect the viscosity by randomizing velocities. When the impact parameter $b$ is comparable to $G m / v^{2}$, roughly the "gravitational radius" of a particle, the scattering angle $\Theta$ covers the full range of possibilities. Thus, if the impact parameter is stochastic, the scattered velocities are as well; close gravitational interactions act like elastic collisions. Then, to derive the spreading of a ring, we may adopt the formalism for collisions described in $\$ 3.3$. Thus, in the optically thin limit, there is an effective viscosity that describes both physical collisions and gravitational scattering. (For rigorous treatments of diffusive spreading by gravity see Ohtsuki \& Tanaka 2003, Ormel et al. 2012 and Glaschke et al. 2014.)

\subsubsection{Collective gravitational effects: migration}

In addition to contributing to random motions, pairwise gravitational interactions also act collectively to produce steady flows. Studies of planetary and satellite migration Lin \& Papaloizou 1979a; Goldreich \& Tremaine 1980; Ward 1997) show how swarms of particles contribute over relatively long distances, $O(10) r_{\mathrm{H}}$ to cause a steady radial drift of mass in the ring.

To quantify this effect, we start with the Rutherford formula (Equation (25)), and work only in the limit of small scattering angle $\Theta$ since we are interested in larger-scale collective effects, not small-scale random motions. As above, we consider two particles with masses $m_{1}$ and $m_{2}$ on approaching trajectories in their center-of-mass frame with impact parameter $b$ and closing speed $v$. When the two particles are both orbiting a central body, scattering through an angle $\Theta$ translates to changes in orbital elements (Lin \& Papaloizou 1979b; Goldreich \& Tremaine 1980). To see how this process plays out, we first consider a pair of particles with extremely unequal masses.

We suppose $m_{1} \rightarrow m$ and $m_{2} \rightarrow \delta m \ll m$ and that the more massive particle is on a circular path around a central object of mass $M$ at an orbital distance $a$. Similarly, the lighter body has an orbital distance of $a+q$, with $q / a \ll 1$, orbiting on a circle in the same sense. We can then write the denominator of equation (25) as a product of the pair's relative orbital speed,

$$
v \approx \Omega a\left(\frac{1}{\sqrt{1+q / a}-1}\right),
$$

and the relative orbital angular momentum

$$
\ell \approx \delta m \Omega a q\left(1+\frac{q}{a}\right)\left[1-\frac{1}{(1+q / a)^{3 / 2}}\right]
$$

where $\Omega$ is the orbital angular velocity of the massive body. The change of angular momen- 
tum of the smaller object is

$$
\delta \ell=(1-\cos \Theta) \ell \approx \frac{1}{2} \Theta^{2} \ell
$$

in this small-angle, "weak scattering" limit. Combining these results and expanding in terms of $q$, the change in orbital angular momentum is

$$
\delta \ell \approx \frac{16}{9} \frac{G^{2} m^{2} \delta m a}{\Omega^{3} q^{5}}
$$

This perturbation of the small satellite causes an equal and opposite change in angular momentum of the larger particles, $\Delta \ell=-\delta \ell$. The semimajor axis of the larger object also changes. For circular orbits,

$$
\ell^{2} \approx G M a
$$

Taking derivatives of both sides of this expression gives the change in the larger body's semimajor axis as

$$
\delta a \approx-\frac{2 \delta \ell}{m \Omega a}
$$

Applying Kepler's third law $\left(G M=\Omega^{2} a^{3}\right)$ :

$$
\delta a \approx-\frac{32}{9} \frac{m \delta m}{M^{2}} \frac{a^{6}}{q^{5}}\left(1+\frac{9}{4} \frac{q}{a}+\ldots\right) \quad[m \gg \delta m]
$$

A similar analysis of scattering between equal mass objects gives

$$
\delta a \approx-\frac{256}{25} \frac{m^{2}}{M^{2}} \frac{a^{6}}{q^{5}}\left(1+\frac{27}{20} \frac{q}{a}+\ldots\right) \quad \text { [equal-mass satellites] }
$$

The change in orbital distance per encounter per unit mass is

$$
\delta a_{m}(a, q) \approx-\frac{8 A m}{M^{2}} \frac{a^{6}}{q^{5}}\left(1+B \frac{q}{a}\right)
$$

where $A=4 / 9$ and $B=9 / 4$ when $m \gg \delta m$, and $A=32 / 25$ and $B=27 / 20$ when the mass of the scatterers is equal. Thus, in general $A=0.4-1.3$ and $B=1.3-2.3$ (the factor of 8 in Equation (36) was chosen to keep these constants near unity).

Equation (36) is valid only for a limited range of orbital configurations. The Hill radius of the larger satellite, which characterizes that particle's domain of influence, provides a lower limit to the orbital separation $q$. Furthermore, for the weak theory to apply, an interaction with separation $q$ must yield small orbital perturbations. This condition is violated if the orbital separation is within

$$
q_{\text {xing }} \equiv 2 \sqrt{3} r_{\mathrm{H}} \approx 3.5 r_{\mathrm{H}}
$$


since the outcome of such encounters is that the pair chaotically cross orbit 1 (Gladman 1993). The theory breaks down at large orbital separations, where the curvature of the satellites' orbits plays a role in the interactions (Bromley \& Kenyon 2011b). Combining these two constraints gives

$$
q_{\text {xing }} \lesssim q \lesssim 0.1 a
$$

which is an approximate condition for Rutherford-style weak scattering.

Migration theory uses these conclusions to predict the drift rate of a satellite in a particle disk by tracking the rate of encounters along with the orbital displacement per encounter (e.g., Ida et al. 2000). This same strategy applies to a ring of equal-mass particles. The interaction rate between pairs of particles at orbital separation $q$ is set by their synodic period:

$$
T_{\mathrm{syn}}(a, q) \approx \frac{2 a T_{\mathrm{K}}}{3|q|[1-5 q /(4 a)]}
$$

Integration over the disk gives the total drift rate,

$$
\dot{a}_{\text {grav }} \approx \int 2 \pi(a+q) d q \mathcal{H}(q) \frac{\Sigma(a+q) \delta a_{m}(a, q)}{T_{\text {syn }}(a, q)}
$$

where $\mathcal{H}(q)$ is a high-pass filter, set to zero at small scales (e.g., $|q| \lesssim 3 r_{\mathrm{H}}$ ) and unity elsewhere. Thus the filter eliminates contributions to the drift rate on scales where the weak-scattering theory breaks down and where interactions are dominated by random motions.

With the weak-scattering result for $\delta a$, and a Taylor expansion in terms of the radial distance variable $q$, the drift rate becomes

$$
\dot{a}_{\text {grav }} \approx-\frac{24 A \pi a^{2} \Sigma}{M} \frac{m}{M} \frac{a}{T} \int \operatorname{sgn}(q) \mathcal{H}(q) \frac{d q}{a} \frac{a^{4}}{q^{4}} \times\left[1+\frac{q}{a}\left(B-\frac{1}{4}+\frac{a}{\Sigma} \frac{d \Sigma}{d a}\right)\right]
$$

where the constants $A$ and $B$ are of order unity and depend on the mass ratio of the drifting particle and other objects in the disk (see Equations (34) and (35) ).

In a dynamically cold ring — where the scale height $h$ is smaller than the Hill radius of the ring particles - the minimum orbital distance between particles for which steady migration is possible is $q_{\text {min }} \approx q_{\text {xing }}$ (Equation (37)). Inside this distance, orbits are chaotic and can cross, enabling physical collisions. Outside this scale, orbital evolution is diffusive. The ring of particles expands until particle separations become very large (e.g., Equation (38)) .

\footnotetext{
${ }^{1}$ For exactly circular orbits in the extreme mass-ratio case, weak deflections can cause orbits to cross when the orbital separation is less than $2 \sqrt{3} r_{\mathrm{H}}$.
} 
If the ring is dynamically hot, with random particle excursions comparable to the scale height $h$, then $q_{\min } \approx h$. By setting $\mathcal{H}(q)=0$ when $|q|<q_{\min }$ and unity elsewhere, we obtain a general expression for the radial migration of a ring particle: The radial drift rate of a particle embedded in the ring is

$$
\dot{a}_{\text {grav }} \approx-\frac{24 \pi a^{2} \Sigma}{M} \frac{m}{M} \frac{a}{T} \frac{a^{2}}{q_{\text {min }}^{2}} A \times\left(B-\frac{1}{4}+\frac{a}{\Sigma} \frac{d \Sigma}{d a}\right)
$$

where

$$
q_{\text {min }}=\max \left(q_{\text {xing }}, h\right) \quad \text { or } \quad q_{\min }^{2} \approx\left(16 r_{\mathrm{H}}^{2}+h^{2}\right) .
$$

The rightmost expression just serves to enforce the minimum value of $q_{\min }$ as a smooth function of $h$ or $r_{\mathrm{H}}$. In the expression for the drift rate itself, the quantity $B-1 / 4$ represents a systematic inflow of geometrical origin in a disk with uniform surface density, while the logarithmic derivative corresponds to a radial flux in a non-uniform disk analogous to diffusion in a viscous ring. Together these two terms define an equilibrium surface density with no radial flux $(d \ln \Sigma / d \ln a=1 / 4-B)$. Near the inner edge of the ring, where the radial gradient of $\Sigma$ is large, material flows inward. Similarly, the ring spreads outward near the outer edge.

\subsection{Ring evolution}

Astrophysical rings continuously evolve with time. Physical collisions and gravitational interactions produce diffusive spreading, growth, and destruction. Inside the Roche limit, simplifying assumptions, such as requiring fixed particle size (Goldreich \& Tremaine 1978; Salmon et al. 2010), allow elegant solutions to this evolution. Here, our goal is to track simultaneous growth and viscous spreading. We take advantage of the idea that pairwise interactions drive all the dynamics. In a steady ring, most interactions involve scattering and are neither destructive or fully inelastic (all collisions lead to perfect mergers). Then, the local dynamics (viscous or gravitational spreading) can be handled by approximating particles as having a fixed size over a certain short time scale to track the dynamical evolution of the ring. Particle growth can then enter as a separate step in the evolution, in staggered fashion, in the spirit of a standard leap-frog technique. This approach also allows us to use standard tools of the trade (Pringle 1981) to calculate the ring evolution. 


\subsubsection{Steady-state theory of ring evolution}

We describe the evolution of a ring in terms of its surface density. The evolution equation follows from conservation of mass and angular momentum (Pringle 1981). Integrated over the direction perpendicular to the ring plane, these conservation laws are

$$
\begin{gathered}
\frac{\partial \Sigma}{\partial t}+\frac{1}{a} \frac{\partial}{\partial a}(a \dot{a} \Sigma)=0 \\
\frac{\partial}{\partial t}\left(a^{2} \Omega \Sigma\right)+\frac{1}{a} \frac{\partial}{\partial a}\left(a^{3} \dot{a} \Omega \Sigma\right)=\frac{1}{2 \pi a} \frac{\partial \dot{L}}{\partial a}
\end{gathered}
$$

where $a$ is orbital distance, $\Omega$ is orbital angular speed at distance $a$, and $\dot{L}$ represents the torque on an annulus of radius $a$ and width $\Delta a$. These equations come from applying the total time derivative operator $d / d t$ in cylindrical coordinates to the total mass and angular momentum associated with the annulus. In the case of angular momentum conservation, the derivative of the torque $\dot{L}$ follows from taking the limit of $\Delta a \rightarrow 0$. Combining these expressions we obtain

$$
a \dot{a} \Sigma=\left[\frac{\partial}{\partial a}\left(a^{2} \Omega\right)\right]^{-1} \frac{1}{2 \pi} \frac{\partial \dot{L}}{\partial a} \approx \frac{1}{\pi a \Omega} \frac{\partial \dot{L}}{\partial a}
$$

where the expression on the right applies in the limit of Keplerian flow. Putting this expression back into the continuity equation gives the evolution equation for the surface density:

$$
\frac{\partial \Sigma}{\partial t}=-\frac{1}{a} \frac{\partial}{\partial a}(a \dot{a} \Sigma)=-\frac{1}{a} \frac{\partial}{\partial a}\left(\frac{1}{\pi a \Omega} \frac{\partial \dot{L}}{\partial a}\right) .
$$

It remains to quantify the torque, either in terms of $\dot{L}$ or the corresponding radial drift $\dot{a}$. Multiple sources of torque are just summed together, using both $\dot{L}$ (for viscosity) and $\dot{a}$ (for gravitational spreading). The latter we include in the form of Equation (41), while the former we calculate next, following Pringle (1981).

The viscosity in an astrophysical ring relates the shear in the velocity flow $(a \partial \Omega / \partial a$, which is $-3 / 2 \Omega$ for a Keplerian ring) to the torque on an annulus,

$$
\dot{L}=\nu a \cdot 2 \pi a \Sigma \cdot a \frac{\delta \Omega}{\delta a} \approx-3 \pi a^{2} \Sigma \nu \Omega
$$

These expressions define the kinematical viscosity $\nu$. They may be easily related to the laboratory definition of dynamical viscosity by specifying a disk scale height and working with the force applied to the common surfaces between an annulus and its neighbors. 
Putting the above result (Equation (48)) together with the expression for the radial drift rate from weak scattering (Equation (42)) yields the desired evolution equation:

$$
\frac{\partial \Sigma}{\partial t}=\frac{1}{a} \frac{\partial}{\partial a}\left[3 a^{1 / 2} \frac{\partial}{\partial a}\left(\nu \Sigma a^{1 / 2}\right)-\left(a \Sigma \dot{a}_{\text {grav }}\right)\right] .
$$

In this expression, the first term on the right-hand-side gives the diffusive evolution of $\Sigma$ from viscosity $\nu$. The second term corresponds to gravitational scattering. Under some circumstances, for example, a ring of monodisperse particles with a shallow surface density gradient and relative speeds higher than the escape velocity, we can derive an approximate viscosity for the gravitational interactions,

$$
\nu_{\mathrm{grav}} \approx \frac{v_{\mathrm{esc}}^{2} \Sigma T_{\mathrm{K}}}{\rho r} \frac{v_{\mathrm{esc}}^{2}}{v^{2}} \quad\left(v>v_{\mathrm{H}}\right) .
$$

This expression is comparable, to within a constant of order unity, to similar expressions derived from the standpoint of kinetic theory (Shu \& Stewart 1985). Since we derive the ring evolution directly from scattering theory, and do not seek a quantity of the form of a viscosity parameter, our theory differs slightly from the kinetic approach. In principle, an advantage of our approach is that it can accommodate migration when the distribution of ring particle mass covers a wide range. Below (\$3.5.2) we further discuss the connection between the evolution from the effective (collisional) viscosity and the contribution from gravity in our formalism.

The solution of Equation (49) requires boundary conditions. In general, the outer edge of the ring might expand into a vacuum (or be truncated artificially at the edge of a computational domain). The inner boundary is more sensitive to the astrophysical setting. With a single central mass, material may be lost by accretion, thus $\Sigma=0$ at its surface. For a circumbinary ring, the lack of stable orbits within a factor of at least twice the binary separation (for comparable-mass binaries) establishes the inner boundary of the disk. Preliminary simulations show that particles at the inner edge get pushed back into the ring, or are trapped in resonances there $(\$ 4.1$, below). We therefore provisionally set inner boundary condition at the innermost stable circular orbit to be reflective, $d \Sigma / d a=0$ (see also Pringle 1991).

Together with specific values for $\nu(\$ 3.3)$ and the drift rate from mutual weak scattering of ring particles (\$3.4), these boundary conditions allow us to track the spreading of a ring from some initial state. 


\subsubsection{Connection between weak scattering and ring viscosity}

Ring viscosity and gravitational scattering have similar physical origins. In both cases, interactions between pairs of particles cause deflections which lead to a radial mass flux. There are also profound differences. Deflections in a viscous ring derive from physical collisions, which can stir particles up until collisional damping becomes important (Cook \& Franklin 1964; Goldreich \& Tremaine 1978). Gravitational scattering can also stir particles, but only until they reach the escape speed. Beyond that speed, gravitational scattering becomes ineffective. Furthermore, gravitational interactions become irrelevant if the particles lie inside the Roche limit. In both of these situations, the ring is completely driven by the viscosity from physical collisions. Otherwise, gravity and viscosity share in the evolution of the ring.

To compare quantitatively how viscosity and gravity operate, we focus on the surface density flux terms (right hand side of Equation (49)). Under a set of simplifying assumptions, including constant viscosity $\nu$ and surface density $\Sigma$ in a ring of monodisperse particles, we write the fluxes in terms of the particle escape velocities $v_{\text {esc }}$ :

$$
\begin{aligned}
J_{\text {visc }} & \equiv \frac{3}{2} \nu \Sigma \sim G \Sigma^{2} r T_{\mathrm{K}} \frac{v^{2}}{v_{\mathrm{esc}}^{2}} \\
J_{\text {grav }} & \equiv a \Sigma \dot{a}_{\text {grav }} \sim G \Sigma^{2} r T_{\mathrm{K}} \frac{v_{\mathrm{esc}}^{2}}{v^{2}+C^{2} v_{\mathrm{H}}^{2}}
\end{aligned}
$$

where $C \sim q_{\text {xing }} / r_{\mathrm{H}} \sim 4$. Thus, the particle velocity, as compared to the escape velocity, delineates the viscous and gravity regimes. Roughly, viscosity operates at $v \gg v_{\text {esc }}$, while gravitational scattering drives the evolution at $v \ll v_{\text {esc }}$.

In a viscous disk, the effective viscosity formally depends on the random velocity with no restrictions except that $v$ must small compared to the local Keplerian velocity. In the gravity dominated regime, the surface density flux has a maximum,

$$
J_{\text {grav }} \sim G \Sigma^{2} r T_{\mathrm{K}} \frac{v_{\mathrm{esc}}^{2}}{16 v_{\mathrm{H}}^{2}} \quad\left(v \ll v_{\mathrm{esc}}\right) .
$$

Physically this limit reflects our assumptions about gravitational interactions: particles with

low relative speeds drifting by one another with impact parameters within $q_{\text {xing }}$ are in the chaotic, orbit crossing regime, or possibly corotating. Here, we assume that these types of orbits do not lead to a net radial drift. In any event, if the speeds are below the escape velocity, then collisional damping also must be operating, keeping the ring dynamically cool on these small scales. 


\subsection{Illustrations of the theory}

To give examples of solutions to the evolution equation (49), we use a finite difference code that divides the surface density into 640 radial bins. (In runs described below, we doubled or quadrupled the number of bins to track the physics of any sharp edges that develop.) The initial surface density has a Gaussian profile in orbital distance, and a fixed total mass. In each run, we choose a particle radius and set the dispersion of the particles to be their escape velocity. These parameters, along with the surface density on the grid and its gradient (derived from finite differences) give the viscosity $\nu_{\text {rad }}$ and $\dot{a}_{\text {grav }}$ needed to evolve $\Sigma$ in a step-wise fashion.

Figure 2 show numerical solutions for the evolution of a ring around a central mass. The curves in the Figure are snapshots of the evolution, corresponding to a viscous ring and to an inviscid ring that spreads from gravity. The two phenomena yield similar behavior in the ring at this delimiting velocity.

For reference, we consider how the theory applies to Saturn's rings. Inside the Roche limit, mutual gravity between small ring particles is irrelevant. Outside this limit, external influences (distant moons) stir the ring particles to speeds that exceed their escape velocity. For example, the A ring consists of small (centimeter- to meter-size) particles, with a scale

height of roughly 10 meters (e.g., Colwell et al. 2009). The orbital frequency is $\Omega \sim 10^{-4} \mathrm{~s}^{-1}$; typical random speeds, $v \sim h \Omega$, are $\sim 0.1 \mathrm{~cm} / \mathrm{s}$. The escape velocity of a centimeter-size particle is roughly $0.001 \mathrm{~cm} / \mathrm{s}$; thus, viscosity dominates in the ring.

In other planetary rings, conditions probably differ. Our focus turns to the PlutoCharon binary where particle masses may have been large enough for gravity to have an impact on ring evolution.

\section{Circumbinary orbital dynamics}

Pluto and Charon, as partners in the solar system's most prominent binary planet, pose an interesting challenge to our understanding of the dynamics of planetary rings and satellites. Circumbinary orbits differ from Keplerian ones in important ways (Heppenheimer 1978; Murray \& Dermott 1999; Lee \& Peale 2006; Leung \& Lee 2013; Bromley \& Kenyon 2015). In this section, we present an overview of these differences. 


\subsection{Orbital stability and resonances}

At distances comparable to the binary separation, $a_{\text {bin }}$, circumbinary orbits are unstable. To help map out regions of instability around a binary, Holman \& Wiegert (1999) numerically estimate a critical distance from the binary center of mass, $a_{\text {crit }}$, that depends on the binary eccentricity $\left(e_{\text {bin }}\right)$ and mass ratio. Beyond $a_{\text {crit }}$, most orbits are stable over many $\left(10^{4}\right)$ dynamical times. Inside $a_{\text {crit }}$, the gravity of the binary destabilizes orbits. Thus, every binary has an inner cavity that is cleared of ring particles. For Pluto-Charon in its presentday orbit $\left(e_{\text {bin }} \approx 0\right)$, this inner cavity lies inside of $a_{\text {crit }} \approx 2.0 a_{\text {bin. }}$. With $a \sim 2.2 a_{\text {bin }}$, the innermost moon Styx is just beyond this critical orbital distance.

Instabilities arise when resonances with the central binary overlap (Wisdom 1980; Lecar et al. 2001). The Holman \& Wiegert (1999) expression for $a_{\text {crit }}$ gives only an approximate location of the outer edge of the central cavity around the binary. Some stable orbits exist inside of $a_{\text {crit }}$; there may be various unstable resonances outside of it. Popova \& Shevchenko (2013) provide an excellent illustration (seea also Wyatt et al. 1999; Pichardo et al. 2008; Doolin \& Blundell 2011).

For the Pluto-Charon binary, with eccentricity near zero, resonance phenomena are not important except near the 3:1 resonance at $\sim 2 a_{\text {bin. }}$. When the binary has a modest eccentricity, $e_{\text {bin }}=0.1$, however, unstable orbits form around the 5:1 commensurability. For other values of $e_{\text {bin }}$, it is important to map the stability orbits for each astrophysical setting, especially for problems which involve massive satellites (e.g. Popova \& Shevchenko 2013; Cheng et al. 2014a).

Figure 3 illustrates the effects of resonances. Initially, a massless satellite orbits outside of the 7:1 commensurability around an expanding circular binary. On its own, the satellite navigates the 7:1-4:1 commensurabilities as binary expansion causes them to sweep past. Near the 3:1 resonance (with $a$ close to $a_{\text {crit }}$ ), however, the satellite is ejected (Fig. 3, left panel).

In contrast, a satellite that experiences sufficient collisional damping and diffusion survives. In Fig. 3 (right panel), a satellite starts in the same orbit but is subjected to diffusion and random walks in a manner consistent with a monodisperse ring of 1-km particles stirred to their escape velocity in a ring with surface density $40 \mathrm{~g} / \mathrm{cm}^{2}$ (see $\$ 5.2$, below). We mimic these effects in the manner of Bromley \& Kenyon (2013, e.g., Equation (21) and related discussion, above); damping comes from a slow and steady adjustment of the osculating Keplerian eccentricity, while diffusion is a sequence of random walks.

With collisional damping and diffusion, satellites in the rings coexist with the destabilizing resonances. Strong damping (many small particles) allows particles to orbit as if the 
instability did not exist; weak damping (fewer, larger particles) and higher binary eccentricity leaves satellites susceptible to ejection. Intermediate levels of damping and diffusion allow particles to become trapped in resonances and to move with them. In the example shown in the left panel of Fig. 3, stable satellites expanding in the 3:1 commensurability require damping times $\lesssim 50$ yr (more than $10^{3}$ satellite orbital periods). When all of the mass in the ring is in objects with radii smaller (larger) than a few $\mathrm{km}$, the time scale for collisional damping (cf. Equation (11) is smaller (larger) than this requirement. Thus, different mixtures of particle sizes lead to different outcomes for the evolution of the Pluto-Charon binary and its satellites.

\subsection{Circumbinary orbits and most circular paths}

Outside the resonances, circumbinary orbits are stable and can be surprisingly circular. Despite the non-Keplerian gravitational potential of the binary, there is a set of orbits analogous to circular paths around a single central mass. This family of 'most circular' orbits consists of nested, circumbinary trajectories that do not cross (Lee \& Peale 2006; Youdin et al. 2012; Leung \& Lee 2013). Even when the orbit of the central binary is eccentric, the most circular paths have eccentric motion characterized by a 'forced' eccentricity,

$e_{\text {force }}$ Heppenheimer 1978; Murray \& Dermott 1999) that preserves the nested and nonintersecting properties.

Particles with random motion about a most circular orbit have a 'free' eccentricity, $e_{\text {free}}$, which serves the same role as the orbital eccentricity for an orbit around a single central mass. Particles with free eccentricity collide with characteristic relative velocities $e_{\text {free }} v_{\mathrm{K}}$. Particles with eccentricity that damps to zero settle on most circular paths with a forced eccentricity.

\subsubsection{Analytical theory of circumbinary orbits}

Most circular orbits appear as part of general circumbinary orbit solutions from analytical theory in the context of the restricted three body problem. Following Lee \& Peale (2006) and Leung \& Lee (2013), we start with the potential

$$
\Phi=-\frac{G M_{p}}{\sqrt{R^{2}+z^{2}+R_{p}^{2}+2 R R_{p} \cos \Delta \phi}}-\frac{G M_{s}}{\sqrt{R^{2}+z^{2}+R_{s}^{2}-2 R R_{s} \cos \Delta \phi}},
$$

where $\Delta \phi$ is the angle between the secondary and the massless satellite (ring particle) in a reference frame with the binary's center of mass at the origin; in this frame, the satellite is 
at radial position $R$ in the plane of the binary, and altitude $z$ above it, while $R_{p}$ and $R_{s}$ are the orbital distances of the primary and secondary, with masses $M_{p}$ and $M_{s}$. When the binary is circular $\left(e_{\mathrm{bin}}=0\right), R_{p}$ and $R_{s}$ are constants.

The strategy of Lee \& Peale (2006) is to expand the potential in terms of the angle cosines in Equation (54): for a circular binary, this expansion is

$$
\Phi \approx \sum_{k=0}^{\infty} \Phi_{0 k} \cos (k \Delta \phi) \quad\left[e_{\text {bin }}=0 ; z=0\right]
$$

where $\Omega_{\text {bin }}$ is the mean motion of the binary $\left(\Omega_{\mathrm{bin}}{ }^{2}=G\left(M_{p}+M_{s}\right) / a_{\mathrm{bin}}{ }^{3}\right)$, and our choice of time $t$ fixes the orbital phase. Each coefficient $\Phi_{0 k}$ may be expressed as a series in terms of $R_{\mathrm{g}} / a_{\mathrm{bin}}$, where $R_{\mathrm{g}}$ is the radius of a guiding center associated with a satellite's orbit. Lee \& Peale (2006) and Bromley \& Kenyon (2015) list leading terms in these expansions.

The next step is to express a satellite's motion as excursions in radial, azimuthal and altitude coordinates $(\delta R, \delta \phi, z)$ about the guiding center at radius $R_{\mathrm{g}}$. The result is a set of equations of motion that can be linearized in the excursion coordinates. When set up in the manner of driven harmonic oscillators, these equations can be solved with standard techniques. The natural frequencies $\kappa_{\mathrm{e}}$ and $\nu_{\mathrm{i}}$ correspond to the free eccentricity and free inclination. The driving frequencies are the synodic frequency and its harmonics, along with the binary's orbital frequency if the binary is eccentric.

This prescription yields expressions for the satellite position in cylindrical coordinates (Lee \& Peale 2006):

$$
\begin{gathered}
R(t)=R_{\mathrm{g}}\left[1-e_{\text {free }} \cos \left(\kappa_{\mathrm{e}} t+\psi_{\mathrm{e}}\right)-e_{\text {force }} \cos \left(\Omega_{\mathrm{g}} t\right)+\sum_{k=1}^{\infty} C_{k} \cos \left(k \omega_{\mathrm{syn}} t\right)+\ldots\right] \\
\phi(t)=\Omega_{\mathrm{g}}\left[t+\frac{2 e_{\text {free }}}{\kappa_{\mathrm{e}}} \sin \left(\kappa_{\mathrm{e}} t+\psi_{\mathrm{e}}\right)+e_{\text {force }} \sin \left(\Omega_{\mathrm{g}} t\right)+\sum_{k=1}^{\infty} \frac{D_{k}}{k \omega_{\mathrm{syn}}} \sin \left(k \omega_{\mathrm{syn}} t\right)+\ldots\right] \\
z(t)=i R_{\mathrm{g}} \cos \left(\nu_{\mathrm{i}} t+\psi_{i}\right),
\end{gathered}
$$

where $\Omega_{\mathrm{g}}$ is the angular speed of the guiding center, $\omega_{\text {syn }} \equiv \Omega_{\mathrm{bin}}-\Omega_{\mathrm{g}}$ is the synodic frequency of the satellite relative to the binary; orbital elements are $e_{\text {force }}, e_{\text {free }}$ and $i$, the forced eccentricity, the free eccentricity and the inclination, respectively, while the phase angles $\psi_{e}$ and $\psi_{i}$ are constants. The coefficients $C_{k}$ and $D_{k}$ are associated with the time-varying part of the binary's potential. Terms not shown depend on the binary eccentricity (see Leung \& Lee 2013 ). In the form given above, the solutions are accurate when $e_{\text {bin }} \lesssim R_{\mathrm{g}} / a_{\text {bin }} \ll 1$. 
To define the parameters in Equations (56)-(58), we start by noting that the orbital frequency of the guiding center comes from the time-average potential $\Phi_{00}$ (Equation (55)):

$$
\left.\Omega_{\mathrm{g}}^{2} \equiv \frac{1}{R_{\mathrm{g}}} \frac{d \Phi_{00}}{d R}\right|_{R_{\mathrm{g}}}=\frac{G M}{R_{\mathrm{g}}{ }^{3}}\left\{1+\frac{\mu}{M}\left[\frac{3}{4} \frac{a_{\mathrm{bin}}{ }^{2}}{{R_{\mathrm{g}}}^{2}}+\frac{45}{64} \frac{\left(M_{p}^{3}+M_{s}^{3}\right)}{M^{3}} \frac{a_{\mathrm{bin}}{ }^{4}}{R_{\mathrm{g}}{ }^{4}}+\ldots\right]\right\}
$$

the square root of which is the mean motion of the ring particle. Its epicyclic frequency is

$$
\left.\kappa_{\mathrm{e}}^{2} \equiv R_{\mathrm{g}} \frac{d \Omega_{\mathrm{g}}{ }^{2}}{d R}\right|_{R_{\mathrm{g}}}-4 \Omega_{\mathrm{g}}{ }^{2}=\frac{G M}{R_{\mathrm{g}}{ }^{3}}\left\{1-\frac{\mu}{M}\left[\frac{3}{4} \frac{a_{\mathrm{bin}^{2}}}{R_{\mathrm{g}}{ }^{2}}+\frac{135}{64} \frac{\left(M_{p}^{3}+M_{s}^{3}\right)}{M^{3}} \frac{a_{\mathrm{bin}}{ }^{4}}{R_{\mathrm{g}}{ }^{4}}+\ldots\right]\right\} .
$$

A similar analysis based on the expansion of the potential in the $z$-direction yields the vertical excursion frequency,

$$
\left.\nu_{\mathrm{i}}^{2} \equiv \frac{1}{z} \frac{d \Phi}{d z}\right|_{z=0, R_{\mathrm{g}}}=\frac{G M}{R_{\mathrm{g}}{ }^{3}}\left\{1+\frac{\mu}{M}\left[\frac{9}{4} \frac{a_{\mathrm{bin}}{ }^{2}}{R_{\mathrm{g}}{ }^{2}}+\frac{225}{64} \frac{\left(M_{p}^{3}+M_{s}^{3}\right)}{M^{3}} \frac{a_{\mathrm{bin}}{ }^{4}}{R_{\mathrm{g}}{ }^{4}}+\ldots\right]\right\} .
$$

Because $\kappa_{\mathrm{e}}$ and $\nu_{\mathrm{i}}$ differ from the mean motion of the satellite, the free eccentricity and inclination have corresponding nodal precession. In the limit that the binary separation goes to zero, both $\kappa_{\mathrm{e}}$ and $\nu_{\mathrm{i}}$ become the Keplerian mean motion. Next, the coefficients for the forcing terms at the synodic frequency are

$$
\begin{aligned}
C_{k} & =\frac{1}{R_{\mathrm{g}}\left(\kappa_{\mathrm{e}}^{2}-k^{2} \omega_{\mathrm{syn}}^{2}\right)}\left[-\frac{d \Phi_{0 k}}{d R}+\frac{2 \Omega_{\mathrm{g}} \Phi_{0 k}}{R_{\mathrm{g}} \omega_{\mathrm{syn}}}\right]_{R_{\mathrm{g}}} \\
D_{k} & =2 C_{k}-\frac{\Phi_{0 k}}{R_{\mathrm{g}}{ }^{2} \Omega_{\mathrm{g}} \omega_{\mathrm{syn}}}
\end{aligned}
$$

in the notation of Lee \& Peale (2006). Finally, we have the forced eccentricity,

$$
e_{\text {force }}=\frac{5}{4} \frac{M_{p}-M_{s}}{M} \frac{a_{\text {bin }}}{R_{\mathrm{g}}} e_{\mathrm{bin}}-\left[\frac{1}{2} \frac{\mu\left(M_{p}-M_{s}\right)}{M^{2}}+\frac{5}{32} \frac{M_{p}^{3}-M_{s}^{3}}{M^{3}}\right] \frac{a_{\mathrm{bin}}{ }^{3}}{r^{3}}+\ldots
$$

(Heppenheimer 1978; Moriwaki \& Nakagawa 2004; Rafikov 2013). Leung \& Lee (2013) include this term $\left(C_{1}^{-}\right.$in their notation), along with terms similar to $C_{k}$ and $D_{k}$ but reduced by a factor of the binary eccentricity.

\subsubsection{Examples}

Most circular paths, with no inclination or free eccentricity, describe the least dissipative trajectories for particles that suffer collisions. Small particles on these paths remain on them indefinitely without colliding. We construct these trajectories using the orbit solutions 
in Equations (56)-(58). Because the fall-off with $a_{\text {bin }} / R_{\mathrm{g}}$ is shallow for orbits that are modestly close to the binary, we also consider numerical search methods. We evolve many trial paths until we find the one that minimizes the radial excursion (e.g., Youdin et al. 2012). This procedure is slow, as it requires numerical orbit integration with each trial. For the integrations, we use a $6^{\text {th }}$-order symplectic time step algorithm in the N-body component of our Orchestra code (Bromley \& Kenyon 2006, 2011a).

Figure 4 provides an illustration of a particle on a most circular orbit around a binary with $e_{\text {bin }}=0$. It shows a direct comparison between the analytical theory and a most circular orbit found by numerically searching for the trajectory with minimum radial excursions about its guiding center. The small radial excursions of a particle on a most circular orbit have a "W" shape profile over a synodic period, showing (i) maxima when the satellite is aligned with the binary and closest to the secondary, (ii) minima when the satellite is perpendicular to the binary axis, and (iii) local maxima when the satellite is closest to the primary.

In the circular binary's frame of reference, the orbit of a satellite is a closed, egg-shaped loop. All such figures are nested. The further away from the binary, the more circular the figure. Particles on most circular orbits traverse these paths on their own synodic period with respect to the binary. While their speeds may be different, their phases align in a way that all neighboring particles get coherently jostled by the binary. None of their paths cross. Pichardo et al. (2005) provide an excellent description of these orbits in terms of 'invariant loops', closed curves that vary in response to the binary's potential (see also Georgakarakos \& Eggl 2015).

If the binary has some eccentricity, most circular orbits are eccentric. Satellites following these paths have some epicyclic motion about the guiding center, with eccentricity set by $e_{\text {force }}$ in Equation (64). Figure 5 provides an illustration, using a small binary eccentricity to allow the radial excursion from the forced eccentricity to roughly match the radial displacements oscillating at the synodic frequency. With zero free eccentricity, the radial excursions $(\sim$ $\left.R_{\mathrm{g}} e_{\text {force }}\right)$ are steady, with no overall change in their maxima over time.

Figure 5 also shows the effect of free eccentricity. Although the free eccentricity has nodal precession, the forced eccentricity does not. Thus, the radial excursions relative to a purely circular orbit about the binary center-of-mass vary continuously, with a period $2 \pi /\left|\Omega_{\mathrm{g}}-\kappa_{\mathrm{e}}\right|$. Adopting a free eccentricity equal to the forced eccentricity for the example in Figure 5, the total eccentricity cycles between zero and $2 e_{\text {force }}$. Thus orbits with no free eccentricity $\left(e_{\text {free }}=0\right)$ have the smallest maximum radial excursion. More importantly, these orbits do not intersect, offering a place in phase-space for many small particles to coexist without colliding with each other. 


\subsection{Summary}

We conclude this section with its main implication for ring dynamics. The family of most circular orbits represents the dynamically cold state of a ring orbiting a central binary. Collisional damping causes particles to settle onto one of these orbits, just as for circular orbits around a single central mass. As long as the ring is not globally self-gravitating, the most circular orbits represent reference frames in which the local evolution of the ring takes place. Significantly, these orbit solutions exchange no net torque with the binary. Thus, the theory for ring dynamics developed for a purely Keplerian potential applies to circumbinary orbits, as long as the guiding centers are at distances where long-term stable orbits are possible.

Here we take advantage of these results by adopting reference frames that track most circular paths. Then we may perform calculations such as solving for the radial evolution of a circumbinary ring (Equation (49) ) simply by setting the orbital distance variable $a$ to the guiding center radius. There are still differences, although they are typically small. For example, the viscosity in Equation (17) depends on the orbital period, which is reduced by a factor of $3 / 8 \times(\mu / M)\left(a_{\text {bin }} / R_{\mathrm{g}}\right)$ in units of the binary separation. At the orbit of Styx, this correction is about $1 \%$.

The strategy of working in most circular frames fails at distances close to binary or at the locations of resonant instabilities. However, the effects of destabilizing multiple resonances (e.g., Wisdom 1980; Lecar et al. 2001) are avoided if particles are collisionally damped. Furthermore, as in Figure 3. damping and diffusion may facilitate resonant trap-

ping and migration (Ward \& Canup 2006), processes that can enable a ring to expand as its central binary evolves. Preliminary estimates suggest that the damping time expected for a sea of $1 \mathrm{~km}$ particles - roughly $10 \mathrm{yr}$ - is more than sufficient to mitigate the effects of the instabilities outside of the 3:1 commensurability with the binary. On their own, significantly larger objects, with radii exceeding $5 \mathrm{~km}$ or so, may not be able to orbitally damp on their own, but instead require a population of smaller bodies to keep them stable.

\section{The Pluto-Charon system}

In this section, we apply the theory of circumbinary ring dynamics and evolution to Pluto, Charon, and a ring where satellites form. Aside from deriving quantitative estimates for time scales and rates, we numerically solve for the evolution of possible ring configurations. We begin with a consideration of the binary itself, since its evolution certainly affects the ring. 


\subsection{Formation and evolution of the binary}

Pluto and Charon have radii $R_{\mathrm{P}} \approx 1153 \mathrm{~km}$ and $R_{\mathrm{C}}=603.6 \mathrm{~km}$ and masses $M_{\mathrm{P}} \approx$ $1.305 \times 10^{25} \mathrm{~g}$ and $M_{\mathrm{C}} \approx 1.520 \times 10^{24} \mathrm{~g}$ (Buie et al. 2006; Person et al. 2006; Tholen et al. 2012; Brozović et al. 2015). Their orbit has separation $a_{\mathrm{PC}} \approx 16.97 R_{\mathrm{P}}$, eccentricity $e_{\mathrm{PC}}$ consistent with zero, and is tidally locked (Buie et al. 2012). The binary orbit relative to the orbital plane of the major planets is significantly inclined, with $i_{\mathrm{PC}} \approx 96^{\circ}$.

An impact origin for the Pluto-Charon is plausible, both in terms of the collision outcomes as compared to the binary's present configuration and the likelihood of such a collision in the outer solar system (McKinnon 1989; Stern 1992; Canup 2005; Kenvon \& Bromley 2014). Simulations (Canup 2005) suggest that as much as $3 \times 10^{21} \mathrm{~g}$ - about $0.02 \%$ of the binary's mass — ends up as debris around the binary. This material initially lies on very eccentric orbits extending from distances of $5-10 R_{\mathrm{P}}$ to $30 R_{\mathrm{P}}$. If the orbits circularize, the debris probably forms a compact ring within 15-30 $R_{\mathrm{P}}$ of the center of mass (Kenyon \& Bromley 2014).

Kenyon \& Bromley (2014) propose that collisional evolution of this ring leads to the formation of the four known satellites and perhaps other, smaller satellites at larger distances from Pluto-Charon. The known satellites - Styx (at an orbital distance of approximately $\left.37 R_{\mathrm{P}}\right)$, Nix $\left(43 R_{\mathrm{P}}\right)$, Kerberos $\left(50 R_{\mathrm{P}}\right)$ and Hydra $\left(57 R_{\mathrm{P}}\right)$ - have a combined mass in the range of $3 \times 10^{19} \mathrm{~g}$ to $3 \times 10^{21} \mathrm{~g}$ (Buie et al. 2006; Tholen et al. 2012; Brozović et al. 2015; Showalter \& Hamilton 2015). The combined mass is consistent with formation from debris left over from a collision which produces the Pluto-Charon binary. The orbital distances require expansion of the ring.

In an impact scenario, Pluto and Charon have an initial orbital separation of

$$
a_{\mathrm{PC}}(t=0) \approx 5 R_{\mathrm{P}}
$$

(Canup 2005). The separation grew in time through exchange of angular momentum that depends on the state of matter within the two partners (e.g., Barr \& Collins 2015); an idealized theoretical rate describing the expansion is

$$
\frac{d a_{\mathrm{PC}}}{d t}=\kappa a_{\mathrm{PC}}^{-11 / 2}
$$

where

$$
\kappa=3 \frac{k}{Q} \frac{M_{\mathrm{C}}}{M_{\mathrm{P}}}\left[\frac{G\left(M_{\mathrm{P}}+M_{\mathrm{C}}\right)}{R_{\mathrm{P}}^{3}}\right]^{1 / 2} R_{\mathrm{P}}^{13 / 2}
$$

with Love number $k=0.058$ and a constant tidal dissipation function $Q=100$ (e.g., Farinella et al. 1979; Dobrovolskis et al. 1997; Peale 1999; Cheng et al. 2014a). The solution 
to these equations thus describes the orbital separation as a function of time:

$$
a_{\mathrm{PC}}(t)=\min \left\{\left[13 \kappa t / 2+a_{\mathrm{PC}}(0)^{13 / 2}\right]^{2 / 13}, 17 R_{\mathrm{P}}\right\}
$$

In this model, the binary expands by a factor of two within $10^{5} \mathrm{yr}$ and reaches its present-day separation within $3 \mathrm{Myr}$, when Pluto and Charon become tidally locked.

The initial eccentricity affects the tidal evolution, typically by speeding up the expansion. However, for modest eccentricity, the effect is weak, and in any event, the eccentricity damps relatively quickly:

$$
\frac{1}{e_{\mathrm{PC}}} \frac{d e_{\mathrm{PC}}(t)}{d t} \approx-\frac{F}{3} \kappa a_{\mathrm{PC}}^{-13 / 2}
$$

where the coefficient $F$ is $O(10)$ (Cheng et al. 2014a). The damping time for an initial eccentricity of order unity in this case is of the order of $1000 \mathrm{yr}$. Hence, we assume that the binary eccentricity $e_{\mathrm{PC}}$ is zero unless otherwise specified.

\subsection{The Pluto-Charon ring: early conditions}

To describe the post-impact conditions in the Pluto-Charon system, we define a fiducial model with a binary separation of $a_{\text {bin }}=5 R_{\mathrm{P}}$, and circumbinary ring radius of $a_{\text {ring }}=20 R_{\mathrm{P}}$. We assume that the mass of the ring is

$$
M_{\text {ring }}=X_{\text {ring }} \cdot 3 \times 10^{20} \mathrm{~g}
$$

where $X_{\text {ring }} \gtrsim 1$ is a rough bound for our models in which the ring must contain enough mass to make the known satellites (Kenyon \& Bromley 2014). We assume that this material has a Gaussian surface density profile with standard deviation of $\Delta a=2 R_{\mathrm{P}}$ :

$$
\begin{aligned}
& \Sigma(t=0)=\Sigma_{G} \exp \left[-\left(a-a_{\text {ring }}\right)^{2} / 2 \Delta a^{2}\right] \\
& \Sigma_{G} \approx 40 X_{\text {ring }}\left[\frac{a_{\text {ring }}}{20 R_{\mathrm{P}}}\right]^{-1}\left[\frac{\Delta a}{2 R_{\mathrm{P}}}\right]^{-1} \mathrm{~g} / \mathrm{cm}^{2} .
\end{aligned}
$$

where $\Sigma_{G}$ is a constant relevant only to this Gaussian model.

To estimate plausible characteristic scales, we consider a monodisperse population of ring particles with $r=1 \mathrm{~km}$ and $\rho=1 \mathrm{~g} / \mathrm{cm}^{3}$, and a characteristic surface density of $40 \mathrm{~g} / \mathrm{cm}^{2}$. The number of particles in the ring is

$$
N \approx 7 \times 10^{4} X_{\text {ring }}\left[\frac{r}{1 \mathrm{~km}}\right]^{-3}
$$


The speeds of individual particles that enter into ring dynamics are

$$
\begin{array}{rrr}
v_{\mathrm{K}}=0.21\left[\frac{a}{20 R_{\mathrm{P}}}\right]^{1 / 2} \mathrm{~km} / \mathrm{s} & \text { [orbital speed] } \\
v_{\mathrm{esc}}=75\left[\frac{r}{1 \mathrm{~km}}\right] \mathrm{cm} / \mathrm{s} & \text { [escape speed] } \\
v_{\mathrm{H}}=16\left[\frac{a}{20 R_{\mathrm{P}}}\right]^{-1 / 2}\left[\frac{r}{1 \mathrm{~km}}\right] \mathrm{cm} / \mathrm{s} & \text { [Hill speed]. }
\end{array}
$$

The latter two speeds are characteristic of gravitational stirring in the ring.

An important consideration for a compact ring is whether it is stable against its own gravity (Toomre 1964). To avoid gravitational clumping of particles within the ring, the particles must maintain a minimum random velocity in addition to their orbital motion. For stability, the characteristic minimum speed is

$$
v_{\mathrm{Q}}=0.94\left[\frac{a}{20 R_{\mathrm{P}}}\right]^{3 / 2}\left[\frac{\Sigma}{40 \mathrm{~g} / \mathrm{cm}^{2}}\right] \mathrm{cm} / \mathrm{s} . \quad \text { [minimum stability speed]. }
$$

In a ring of identical icy particles, this condition translates to a radius of about 10 meters if they stir each other to their escape speed. Since the ring expands to larger radii and lower surface densities, the minimum speed and radius both fall as the ring evolves. Furthermore, if the impact debris contains larger embedded fragments, it will settle to higher speeds, comparable to the escape velocity of those fragments. Thus the bulk of the ring material can be composed of smaller particles and yet the ring will remain gravitationally stable.

In addition to the binary separation and the orbital radius of the ring, there are several other useful length scales:

$$
\begin{array}{ccc}
a_{\mathrm{R}} \approx 3700 \mathrm{~km} & \approx 3 R_{p} & \text { [Roche limit] } \\
r_{\mathrm{H}}=11\left[\frac{a}{20 R_{\mathrm{P}}}\right]\left[\frac{r}{1 \mathrm{~km}}\right] \mathrm{km} & \text { [Hill radius] } \\
h=84\left[\frac{a}{20 R_{\mathrm{P}}}\right]^{3 / 2}\left[\frac{v}{75 \mathrm{~cm} / \mathrm{s}}\right] \mathrm{km} & \text { [ring scale height] }
\end{array}
$$

Finally, results from $§ 2$ provide relevant time scales. Dynamical times are

$$
\begin{aligned}
T_{\mathrm{PC}} & =1.0\left[\frac{a_{\mathrm{PC}}}{5 R_{\mathrm{P}}}\right]^{3 / 2} \mathrm{~d}[\text { binary orbital period }] \\
T_{\mathrm{K}} & =8.2\left[\frac{a}{20 R_{\mathrm{P}}}\right]^{3 / 2} \mathrm{~d}[\text { ring orbital period }]
\end{aligned}
$$


Ring evolution time scales are

$$
\begin{aligned}
T_{\text {damp }} & =14\left[\frac{a}{20 R_{\mathrm{P}}}\right]^{3 / 2}\left[\frac{r}{1 \mathrm{~km}}\right]\left[\frac{\bar{f}_{\text {loss }}}{0.6}\right]\left[\frac{\Sigma}{40 \mathrm{~g} / \mathrm{cm}^{2}}\right]^{-1} \mathrm{yr} \\
T_{\text {migrate }} & \gtrsim 180,000\left[\frac{a}{20 R_{\mathrm{P}}}\right]^{-1 / 2}\left[\frac{r}{1 \mathrm{~km}}\right]^{-1}\left[\frac{\Sigma}{40 \mathrm{~g} / \mathrm{cm}^{2}}\right]^{-1} \mathrm{yr} \\
T_{\text {spread }} & \sim 450,000\left[\frac{a}{20 R_{\mathrm{P}}}\right]^{1 / 2}\left[\frac{r}{1 \mathrm{~km}}\right]^{-1}\left[\frac{\Sigma}{40 \mathrm{~g} / \mathrm{cm}^{2}}\right]^{-1}\left[\frac{v}{v_{\text {esc }}}\right]^{-2} \mathrm{yr},
\end{aligned}
$$

where the migration time $T_{\text {migrate }} \sim a / \dot{a}_{\text {grav }}$, and $T_{\text {spread }}$ is $(a / 2)^{2} / \nu$, giving a rough time scale for the ring to double its size by viscous diffusion. The gravitational spreading time scale is a crude estimate in the limit of a dynamically cold disk; dynamically hot rings, with scale heights that greatly exceed the particle Hill radii, will experience slower evolution by gravitational scattering. The numerical value for the viscous diffusion time carries the assumption that the random component of the particle velocities (governing the ring viscosity) are given by the escape velocity from the particle surface. The surface density $\Sigma$ refers to the local surface density in the ring.

For a ring of monodisperse particles with random velocities comparable to particle escape velocities, damping is generally much faster than spreading. For small particles $(r<1 \mathrm{~km})$ in low-mass rings $\left(\Sigma<40 \mathrm{~g} / \mathrm{cm}^{2}\right)$, evolution time scales are comparable to the expansion time of the binary $\left(10^{5}-10^{6} \mathrm{yr}\right)$. Large particles or large ring mass can shorten the time for the ring to spread. However, it is hard to reduce the spreading time significantly unless particle sizes reach 50-100 km. The small number of ring particles then makes spreading unlikely (see also Peale et al. 2011; Cheng et al. 2014b).

If the ring consists of particles with a broad range of sizes, viscous diffusion can drive a rapid expansion of the ring. If a few large particles (radius $r_{\text {large }}$ ) are embedded in a sea of small ones (radius $r_{\text {small }}$ ), gravitational stirring by the large particles drives the random velocities of the smaller particles to the escape velocity of the large particles. If the large particles have sufficiently large escape velocities, the formal spreading time is very short (Goldreich et al. 2004):

$$
T_{\text {spread }} \sim 5\left[\frac{a}{20 R_{\mathrm{P}}}\right]^{1 / 2}\left[\frac{r_{\text {small }}}{1 \mathrm{~m}}\right]\left[\frac{r_{\text {large }}}{10 \mathrm{~km}}\right]^{-2}\left[\frac{\Sigma}{40 \mathrm{~g} / \mathrm{cm}^{2}}\right]^{-1} \mathrm{yr},
$$

This expression assumes that the rate of collisional damping for the small particles is small compared to the stirring rate of the large particles. For a swarm of $1 \mathrm{~m}$ particles, the likely damping time of $0.01 \mathrm{yr}$ (Equation (83) ) is smaller than this spreading time. However, rapid damping promotes the growth of small objects into larger ones (e.g., 
Kenyon \& Bromley 2014), slowing the rate of damping and accelerating the rate of spreading. Because the damping time grows much faster with $a$ than the spreading time, it seems plausible that a swarm of particles with sizes ranging from $1 \mathrm{~m}$ to $1-10 \mathrm{~km}$ could spread radially on time scales ranging from a century to many millenia.

While a two-population model for the ring is simplistic, it captures the relevant physics and has been used successfully in theories for planet formation in circumstellar disks (e.g., Wetherill \& Stewart 1989; Ida \& Makino 1993; Goldreich et al. 2004). For Pluto-Charon, the early evolution of the debris is likely dominated by destructive collisions of particles on very eccentric orbits. For typical collision velocities of $0.1-0.2 \mathrm{~km} / \mathrm{s}$ (Equation (74)), center-of-mass collision energies of $10^{7}-10^{8} \mathrm{erg} / \mathrm{g}$ destroy particles with $r \lesssim 5-10 \mathrm{~km}$ (Benz \& Asphaug 1999; Leinhardt \& Stewart 2012; Kenyon \& Bromley 2014). Large selfgravity saves larger particles from significant mass loss. Rapid collisional damping likely saves much smaller particles, $r \lesssim 1-10 \mathrm{~m}$, from destruction (Kenyon \& Bromley 2014). Fragmentation likely converts intermediate-mass particles, $1-10 \mathrm{~m} \lesssim r \lesssim 5-10 \mathrm{~km}$, into smaller particles which damp rapidly. Thus, two populations of particles - small particles with most of the mass and a few large particles with significant self-gravity — are plausible components of a circularized ring of particles around Pluto-Charon.

\subsection{Evolution of the Pluto-Charon ring}

To investigate the evolution of monodisperse and two-population ensembles of particles in a ring orbiting Pluto-Charon, we solve the radial diffusion equation (Equation (49)) using a finite difference code (see also Bromley \& Kenyon 2011a). The code tracks the surface density $\Sigma$ in $O\left(10^{3}\right)$ radial bins spanning $a=5 R_{\mathrm{P}}$ out to $120 R_{\mathrm{P}}$. In each bin, we calculate the viscosity (Equation (17)) and the radial mass flux from gravitational scattering (Equation (42)). For each time step, the finite difference code updates the surface density from the viscosity and radial mass flux. Tests on standard problems (e.g., Lynden-Bell \& Pringle 1974; Bromley \& Kenyon 2011a) verify the accuracy of our solutions to better than $1 \%$ over $10^{6}-10^{7} \mathrm{yr}$.

In applying our code to the circumbinary environment, we adopt the results of $\&$. Damping and stirring of particles, along with radial transport, take place relative to most circular orbits. Hence, our use of orbital distance $a$ is synonymous with most circular orbit positions characterized by the orbital radius of the guiding center $R_{g}$. We also assume that resonant excitations and instabilities within the ring do not contribute significantly to the evolution. In our fiducial ring with $1 \mathrm{~km}$ particles, the damping time is rapid enough to suppress resonant-driven excitations. These idealizations still allow us to reach our goal of 
understanding how the ring might spread as the central binary expands.

To establish the range of possibilities for the expansion of a compact ring around a young Pluto-Charon, we choose from a palette of models to test the effects of particle size $(r)$, velocity $(v)$, and total mass in the ring $\left(M_{\text {ring }}\right)$. Our initial conditions assume a Gaussian surface density profile (Equation (71)) with $a=20 R_{\mathrm{P}}, \Delta a=2 R_{\mathrm{P}}$. We start the binary at an orbital separation of $5 R_{\mathrm{P}}$, and have it expand on a time-scale of $\sim 10^{5} \mathrm{yr}$, according to Equation (68).

An additional model parameter is the choice of the inner boundary condition. We can set the inner boundary to be "flow-through" under the assumption that all material reaching the inner edge is lost, presumably to ejection or accretion by the binary (e.g., Pringle 1991). However, in most of our trials, the inner boundary is reflective at the edge of the stability zone. We enforce this boundary by setting up an artificial outflow that is constant inside the ring edge and falls off exponentially over a scale length of $2.5 \%$ of the orbital distance of the edge. This algorithm prevents a strong discontinuity from building up at the inner edge of the ring. Even with this approach, the gradient at the edge can be large and can lead to numerical instability. Thus, we add artificial viscosity in the vicinity of the edge in cases where gravitational scattering strongly dominates over viscous spreading.

For all models we set $\Sigma$ to zero at the outer boundary, although in most cases the ring never reaches the outer edge of the computational domain.

The following list summarizes our models. We use the approximate time for the ring to reach $60 R_{\mathrm{P}}$, just beyond the present-day orbit of Hydra, as a benchmark. If this time is within $\sim 10^{4} \mathrm{yr}$, then the ring may spread before large satellites grow within it (Kenyon \& Bromley 2014). Otherwise, some evolution of particle size — or some other means of migration — is required as the ring expands.

- Baseline model. Our starting point is a ring with total mass $3 \times 10^{20} \mathrm{~g}\left(X_{\text {ring }}=1\right)$ made up of kilometer-size particles stirred to their escape speed ( $\sim 75 \mathrm{~cm} / \mathrm{s})$. Figure 6 illustrates the spreading of this ring. This ring spreads slowly compared to satellite growth times, extending past $60 R_{\mathrm{P}}$ in a few times $10^{5} \mathrm{yr}$.

- Massive rings. If the ring is more massive than the baseline model, the spreading time is reduced $\left(T_{\text {spread }} \sim 1 / \Sigma \sim 1 / X_{\text {ring }}\right)$. Figure 7 gives an example for a ring with $M_{\text {ring }}=3 \times 10^{21} \mathrm{~g}\left(X_{\text {ring }}=10\right)$. In this model, the ring reaches $40 R_{\mathrm{P}}$ in a $10^{4} \mathrm{yr}$ and is beyond $70 R_{\mathrm{P}}$ by $10^{5} \mathrm{yr}$. Compared to the estimated growth time scale of $10^{3}-10^{4} \mathrm{yr}$, this expansion rate is still slow.

- Massive rings with loss through the inner boundary. In previous models, we use re- 
flective inner boundaries, since material at the inner edge is presumably pushed back into the ring by the binary. Here we explore the possibility that mass instead flows through the inner edge and is ejected or accreted by the binary. Figure 8 shows an example. It shows the baseline model, as well as a model with a ring that is ten times more massive, both with and without loss of mass through the inner boundary. The effect of the mass leakage is a loss of about $20 \%$ of the total mass, a flattening of the surface density profile, and a slightly less extended disk.

- Growth of particles. We start with the baseline model, but with particles of radius $r_{\min }=10 \mathrm{~m}$. These particles grow monotonically to a maximum radius of $r_{\max }=3 \mathrm{~km}$ on a time scale of $\tau_{\text {grow }}$ according to

$$
r(t)=r_{\min }+\left(r_{\max }-r_{\min }\right)\left[1-\exp \left(-t / \tau_{\text {grow }}\right)\right]
$$

The random velocity is set to be the instantaneous escape speed of the particles. Figure 9 shows the result. It differs from the baseline model because the spreading starts off more slowly and becomes more rapid only at the late stages, as $r$ grows. Even so, the ring extends to only $50 R_{p}$ in $10^{5} \mathrm{yr}$.

- Stirring by embedded satellites. Guided by the physics of planet formation (Kenyon \& Bromley 2004), we finally consider a scenario in which a population of small ( $r=1 \mathrm{~m}$ ) particles is stirred by a few larger ones $(r=10 \mathrm{~km})$. We include the effect of these larger bodies by stirring the smaller ones to a random speed of half of the Hill velocity of $10 \mathrm{~km}$ objects (fixed at its value at $a=20 R_{\mathrm{P}}$ ). This choice acknowledges that the larger bodies are rare, and that damping among the smaller bodies prevents them from being stirred to the escape speed of the embedded satellites. The result is presented in Figure 10, The ring expands to Hydra's present-day orbit in just over $1000 \mathrm{yr}$, and by $10^{4} \mathrm{yr}$, it has spread to well over $100 R_{\mathrm{P}}$.

To summarize our results, monodisperse populations of ring particles stirred to their own escape velocity, have a spreading time that decreases with particle radius and mass of the ring (Equation (85)). For particles with radii larger than a few kilometers and for ring masses larger than $\sim 10^{21} \mathrm{~g}$, the ring spreads to Hydra's orbit within $10^{5} \mathrm{yr}$. Allowing for particle size to grow from some small value $(r \sim 10 \mathrm{~m})$ only delays the spreading.

In models inspired by planet-forming disks, with small particles and embedded satellites, the ring can spread quickly (Equation $(\underline{86})$ ). Even a low mass ring $\left(3 \times 10^{20} \mathrm{~g}\right)$ can spread out past Hydra on a time scale of $\sim 1000 \mathrm{yr}$. A weakness of this scenario is that the stirring is kept at a constant level throughout the ring as it spreads. For this scenario to be possible, embedded satellites must either migrate within the disk, or form in the outer regions of the 
disk as it spreads. This latter possibility seems plausible, since formation times can be short if the ring should dynamically cool by collisional damping (Kenyon \& Bromley 2014).

\section{Conclusions}

We consider the evolution of a ring of particles around a central binary, with a focus on the Pluto-Charon system. Compared to earlier theories for evolving disks of particles (e.g., Cook \& Franklin 1964; Goldreich \& Tremaine 1978), we add two new features: (i) gravitational scattering, which we quantify in terms of weak scattering theory, and (ii) the impact of the circumbinary environment on ring evolution. Gravitational scattering by massive particles can contribute significantly to the expansion of the ring. As for the circumbinary environment, aside from defining an orbital plane and creating an inner edge by destabilizing orbits (Holman \& Wiegert 1999), the impact of the binary is surprisingly small.

Our main results are listed here, followed by a summary of our conclusions.

- Ring evolution. Our analysis of weak gravitational scattering provides a natural extension to the viscous evolution of particle rings and disks. The expressions of Cook \& Franklin (1964) and Goldreich \& Tremaine (1978) hold until gravitational interactions start to become important. The demarcation between the viscous and gravity regimes is the ratio of the random speeds of particles to their typical escape speed. If the random speeds are large relative to $v_{\text {esc }}$, the particles do not feel their mutual gravity - evolution is viscous. When particles orbit beyond the Roche limit and have random speeds comparable to or smaller than their escape velocities, they stir one another gravitationally. Our results allow a smooth transition between these two regimes.

- Most circular orbits. The circumbinary environment regulates the inner edge of a ring and can destabilize orbits near close-in resonances (e.g.,Wisdom 1980; Murray \& Dermott 1999; Pichardo et al. 2008; Popova \& Shevchenko 2013). Although the binary's time varying gravitational potential modifies orbits in the rest of the ring, the family of most circular orbits provides reference frames similar to the circular orbits around a single central mass (Lee \& Peale 2006; Leung \& Lee 2013). Dissipative disks or rings settle on to these orbits, and perturbations (e.g., from gravitational stirring) cause particles to acquire free eccentricity and inclination relative to these frames. Thus, we can calculate ring evolution in the same way around a binary as we do around a single planet or star (Bromley \& Kenyon 2015). 
- Secular excitations. In initiating the research reported here, our expectation was that stirring by the binary might help the viscous spreading of the ring (e.g., Moriwaki \& Nakagawa 2004; Paardekooper et al. 2012; Rafikov 2013; Lines et al. 2014). However, collisional damping causes particles to settle on most circular paths, even in the presence of a massive axisymmetric disk and distant massive perturbers. Thus, the central binary is not necessarily a source of stirring or torque exchange. We explore the implications for the early stages of circumbinary planet formation in a separate paper (Bromley \& Kenyon 2015).

- Resonance effects and instabilities. The binary can still make a mess of orbits. In units of the binary separation, the Pluto-Charon satellites are closer in than known circumbinary planets from NASA's Kepler mission (e.g. Doyle et al. 2011; Welsh et al. 2012; Orosz et al. 2012a, b) . And, unlike most of the exoplanets, the Pluto-Charon moons orbit inside of suspected resonance-driven instabilities. If the moons emerge early in a compact ring, then they can get caught up in resonant orbits that grow along with the tidally evolving binary (Ward \& Canup 2006). While they may be transported in the resonances, they may just as easily get ejected, as our own simple experiments demonstrate (Figure 3). For the Pluto-Charon system, coordinating resonance transport in a way that preserves all of the moons does not seems possible (Cheng et al. 2014a).

- Collisional damping can stabilize resonant orbits. Our simple simulations also demonstrate how collisional damping and diffusion can protect satellite orbits from ejection (see also Ward \& Canup 2006; Lithwick \& Wu 2008; Cheng et al. 2014a). Thus a ring of small particles can survive the sweep of resonances during tidal expansion. Similarly, as long as a substantial reservoir of small particles is in place, we speculate that between collisional damping among the small objects and dynamical friction between these small bodies and the large ones, the entire population is protected agains unstable resonances.

- Evidence for resonant migration. Our simple simulations also demonstrate how collisional damping and diffusion can facilitate resonant migration (Ward \& Canup 2006). Small particles become trapped near resonances and then get swept up in them as the binary expands. Figure 3 illustrates the phenomenon and highlights the role of binary eccentricity in the process. If a reservoir of small particles is maintained during the binary's tidal evolution, small moons might also participate in this migration. It is beyond our scope here to explore these possibilities in detail, but our anecdotal results are promising. We plan more detailed simulations to test this possibility. 
On the basis of these ideas and our numerical simulations, we infer the following conclusions for the Pluto-Charon system:

- Starting with a compact ring around Pluto-Charon with mass comparable to the observed moons, viscosity and gravity can spread the ring to the moons' present locations in less than $10^{5}-10^{6} \mathrm{yr}$. These time scales are comparable to the tidal evolution of the binary's semimajor axis, but are substantially longer than the growth time of $10-100 \mathrm{~km}$ satellites $\left(\sim 10^{3} \mathrm{yr}\right)$.

- The spreading of the ring can be comparable with, or faster than, the growth time if (i) the ring has small (1-meter) particles stirred by large $(1-10 \mathrm{~km})$ bodies, or (ii) the mass of the ring is large $\left(\gtrsim 10^{22} \mathrm{~g}\right)$. In scenario (i) large objects can probably form as the ring spreads. In both scenarios, the ring spreads quickly to distances well beyond Hydra, the outermost known moon.

- Resonance-driven instabilities remove isolated satellites or moons (e.g., Cheng et al. 2014a). This phenomenon is complicated by the tidal evolution of Pluto-Charon, which causes commensurabilities to sweep through the system. However, collisional damping and diffusion can make satellites immune to resonance-driven instabilities and can also facilitate trapping in a resonance. In the latter case, ring material can be transported outward along with the resonances, as envisioned for full-fledged moons by Ward \& Canup (2006).

- Together, the spreading mechanisms and interactions with resonances suggest potential growth modes for the satellites. In situ formation can occur quickly $\left(10^{3} \mathrm{yr}\right.$; Kenyon \& Bromley 2014); the time needed for spreading the ring can be comparably fast or slow $\left(10^{5} \mathrm{yr}\right)$, leading to these possibilities:

i. The spreading might occur before the expansion of the binary. Then the sweep of resonances might eject or collisionally destroy the largest satellites. They would have to re-emerge out of the debris once the sweep was complete. This scenario would require a massive initial ring, as particles could be lost in the process. (Kenyon et al. 2014 describe a similar picture for Fomalhaut b.) Simulations suggest that there was enough mass in the ring initially to sustain a mass loss of over 99\% and still have enough to make the moons (Canup 2005, 2011). The result could be an extended disk and small as-yet-undetected moons beyond Hydra (Kenyon \& Bromley 2014).

ii. If the ring spreads slowly, then particles in it could get caught up in one or more resonances with the expanding binary. In situ formation during the expansion 
is possible, but a reservoir of small particles (perhaps replenished by collisions) would be essential to prevent the instabilities from leading to ejection. Preliminary numerical experiments (Figure 3) show that material might be trapped in several different commensurabilities, depending on the binary eccentricity and the particle damping/diffusion.

The next step is to incorporate the physics of ring spreading into Orchestra, our hybrid N-body-coagulation code (Bromley \& Kenyon 2011a). Then, we can track the detailed interplay between ring spreading and satellite growth. The N-body part will be essential to simulate resonant-driven instabilities that can clear out orbits in the ring (Cheng et al. $2014 \mathrm{~b})$. How these instabilities play out will depend on a number of factors including the effectiveness of orbital damping, and the time-varying eccentricity of the binary, since circularization brings some stability (Holman \& Wiegert 1999). Our code will enable us to determine how collisional damping, gravitational stirring, resonance-driven instabilities, ring spreading and tidal evolution can work in concert to make the binary's intricate system of moons.

Meanwhile, our models suggest that the expansion of a narrow ring around the PlutoCharon binary is plausible. The extent of the expansion depends on the details. The presence of small moons beyond Hydra's orbits would suggest a more massive disk with small particles stirred by larger ones. Then, we expect 3-5 satellites with radii of a kilometer or so, on circular orbits beyond $60 R_{\mathrm{P}}$ (Kenyon \& Bromley 2014). Ring evolution models with less mass and less stirring leave the ring truncated at Hydra's orbit. Even so, scattering by the growing moons could place some satellites beyond Hydra, although their eccentricity is expected to be significant. New Horizons, with its unprecedented view of the Pluto-Charon satellite system, will test these scenarios.

We are grateful for the comments of an anonymous referee that resulted in a number of improvements to our presentation. We thank NASA for support through the Astrophysics Theory and Origins of Solar Systems programs (grant NNX10AF35G) and through the Outer Planets Program (grant NNX11AM37G). We are also grateful to NASA for a generous allotment of supercomputer time on the 'discover' cluster.

\section{REFERENCES}

Barge, P., \& Pellat, R. 1990, Icarus, 85, 481

Barr, A. C., \& Collins, G. C. 2015, Icarus, 246, 146 
Benz, W., \& Asphaug, E. 1999, Icarus, 142, 5

Brahic, A. 1976, Journal of Computational Physics, 22, 171

Bridges, F. G., Hatzes, A., \& Lin, D. N. C. 1984, Nature, 309, 333

Bromley, B. C., \& Kenyon, S. J. 2006, AJ, 131, 2737

—. 2011a, ApJ, 731, 101

-. 2011b, ApJ, 735, 29

-. 2013, ApJ, 764, 192

—. 2015, ArXiv e-prints, arXiv:1503.03876

Brozović, M., Showalter, M. R., Jacobson, R. A., \& Buie, M. W. 2015, Icarus, 246, 317

Brucker, M. J., Grundy, W. M., Stansberry, J. A., et al. 2009, Icarus, 201, 284

Buie, M. W., Grundy, W. M., \& Tholen, D. J. 2013, AJ, 146, 152

Buie, M. W., Grundy, W. M., Young, E. F., Young, L. A., \& Stern, S. A. 2006, AJ, 132, 290

Buie, M. W., Tholen, D. J., \& Grundy, W. M. 2012, AJ, 144, 15

Canup, R. M. 2005, Science, 307, 546

-. 2011, AJ, 141, 35

Canup, R. M., \& Esposito, L. W. 1995, Icarus, 113, 331

Canup, R. M., \& Ward, W. R. 2002, AJ, 124, 3404

Charnoz, S., Dones, L., Esposito, L. W., Estrada, P. R., \& Hedman, M. M. 2009, in Saturn from Cassini-Huygens, ed. M. K. Dougherty, L. W. Esposito, \& S. M. Krimigis (Dordrecht: Springer Science \& Business Media), 537

Charnoz, S., Salmon, J., \& Crida, A. 2010, Nature, 465, 752

Charnoz, S., Crida, A., Castillo-Rogez, J. C., et al. 2011, Icarus, 216, 535

Cheng, W. H., Lee, M. H., \& Peale, S. J. 2014a, Icarus, 233, 242

Cheng, W. H., Peale, S. J., \& Lee, M. H. 2014b, Icarus, 241, 180

Chiang, E., \& Youdin, A. N. 2010, Annual Review of Earth and Planetary Sciences, 38, 493 
Christy, J. W., \& Harrington, R. S. 1978, AJ, 83, 1005

Colwell, J. E., Nicholson, P. D., Tiscareno, M. S., et al. 2009, in Saturn from Cassini-Huygens, ed. M. K. Dougherty, L. W. Esposito, \& S. M. Krimigis, 375

Cook, A. F., \& Franklin, F. A. 1964, AJ, 69, 173

Dobrovolskis, A. R., Peale, S. J., \& Harris, A. W. 1997, in Pluto and Charon, ed. S. A. Stern \& D. J. Tholen, 159

Doolin, S., \& Blundell, K. M. 2011, MNRAS, 418, 2656

Doyle, L. R., Carter, J. A., Fabrycky, D. C., et al. 2011, Science, 333, 1602

Farinella, P., Milani, A., Nobili, A. M., \& Valsecchi, G. B. 1979, Moon and Planets, 20, 415

Georgakarakos, N., \& Eggl, S. 2015, ApJ, 802, 94

Gladman, B. 1993, Icarus, 106, 247

Glaschke, P., Amaro-Seoane, P., \& Spurzem, R. 2014, MNRAS, 445, 3620

Goldreich, P., Lithwick, Y., \& Sari, R. 2004, ARA\&A, 42, 549

Goldreich, P., \& Tremaine, S. 1980, ApJ, 241, 425

-. 1982, ARA\&A, 20, 249

Goldreich, P., \& Tremaine, S. D. 1978, Icarus, 34, 227

Greenzweig, Y., \& Lissauer, J. J. 1990, Icarus, 87, 40

Heppenheimer, T. A. 1978, A\&A, 65, 421

Holman, M. J., \& Wiegert, P. A. 1999, AJ, 117, 621

Holsapple, K. A., \& Michel, P. 2006, Icarus, 183, 331

-. 2008, Icarus, 193, 283

Hornung, P., Pellat, R., \& Barge, P. 1985, Icarus, 64, 295

Ida, S., Bryden, G., Lin, D. N. C., \& Tanaka, H. 2000, ApJ, 534, 428

Ida, S., \& Makino, J. 1993, Icarus, 106, 210

Jeffreys, H. 1947, MNRAS, 107, 263 
Kenyon, S. J., \& Bromley, B. C. 2001, AJ, 121, 538

—. 2004, AJ, 127, 513

-. 2014, AJ, 147,8

Kenyon, S. J., Currie, T., \& Bromley, B. C. 2014, ApJ, 786, 70

Kenyon, S. J., \& Luu, J. X. 1998, AJ, 115, 2136

Lecar, M., Franklin, F. A., Holman, M. J., \& Murray, N. J. 2001, ARA\&A, 39, 581

Lee, M. H., \& Peale, S. J. 2006, Icarus, 184, 573

Leinhardt, Z. M., \& Stewart, S. T. 2012, ApJ, 745, 79

Leung, G. C. K., \& Lee, M. H. 2013, ApJ, 763, 107

Lin, D. N. C., \& Papaloizou, J. 1979a, MNRAS, 188, 191

-. 1979b, MNRAS, 186, 799

Lines, S., Leinhardt, Z. M., Paardekooper, S., Baruteau, C., \& Thebault, P. 2014, ApJ, 782, L11

Lissauer, J. J. 1987, Icarus, 69, 249

Lithwick, Y., \& Wu, Y. 2008, ArXiv e-prints, arXiv:0802.2951

Lunine, J. I., \& Stevenson, D. J. 1982, Icarus, 52, 14

Lynden-Bell, D., \& Pringle, J. E. 1974, MNRAS, 168, 603

McKinnon, W. B. 1989, ApJ, 344, L41

Meyer-Vernet, N., \& Sicardy, B. 1987, Icarus, 69, 157

Moriwaki, K., \& Nakagawa, Y. 2004, ApJ, 609, 1065

Mosqueira, I., Estrada, P., \& Turrini, D. 2010, Space Sci. Rev., 153, 431

Mosqueira, I., \& Estrada, P. R. 2003a, Icarus, 163, 198

—. 2003b, Icarus, 163, 232

Murray, C. D., \& Dermott, S. F. 1999, Solar system dynamics (Princeton: Princeton University Press) 
Musielak, Z. E., Cuntz, M., Marshall, E. A., \& Stuit, T. D. 2005, A\&A, 434, 355

Ogihara, M., \& Ida, S. 2012, ApJ, 753, 60

Ohtsuki, K. 1992, Icarus, 98, 20

-. 1999, Icarus, 137, 152

Ohtsuki, K., Stewart, G. R., \& Ida, S. 2002, Icarus, 155, 436

Ohtsuki, K., \& Tanaka, H. 2003, Icarus, 162, 47

Ormel, C. W., Ida, S., \& Tanaka, H. 2012, ApJ, 758, 80

Orosz, J. A., Welsh, W. F., Carter, J. A., et al. 2012a, Science, 337, 1511

-. 2012b, ApJ, 758, 87

Paardekooper, S.-J., Leinhardt, Z. M., Thébault, P., \& Baruteau, C. 2012, ApJ, 754, L16

Peale, S. J. 1999, ARA\&A, 37, 533

Peale, S. J., Cheng, W. H., \& Lee, M. H. 2011, in EPSC-DPS Joint Meeting 2011, 665

Person, M. J., Elliot, J. L., Gulbis, A. A. S., et al. 2006, AJ, 132, 1575

Pichardo, B., Sparke, L. S., \& Aguilar, L. A. 2005, MNRAS, 359, 521

-. 2008, MNRAS, 391, 815

Pierens, A., \& Nelson, R. P. 2007, A\&A, 472, 993

Popova, E. A., \& Shevchenko, I. I. 2013, ApJ, 769, 152

Porco, C. C., Thomas, P. C., Weiss, J. W., \& Richardson, D. C. 2007, Science, 318, 1602

Porco, C. C., Weiss, J. W., Richardson, D. C., et al. 2008, AJ, 136, 2172

Pringle, J. E. 1981, ARA\&A, 19, 137

-. 1991, MNRAS, 248, 754

Quintana, E. V., \& Lissauer, J. J. 2006, Icarus, 185, 1

Rafikov, R. R. 2013, ApJ, 764, L16

Rosenblatt, P., \& Charnoz, S. 2012, Icarus, 221, 806 
Safronov, V. S. 1969, Evoliutsiia doplanetnogo oblaka. (Evolution of the Protoplanetary Cloud and Formation of the Earth and Planets, Nauka, Moscow [Translation 1972, NASA TT F-677] (1969.)

Salmon, J., Charnoz, S., Crida, A., \& Brahic, A. 2010, Icarus, 209, 771

Sasaki, T., Stewart, G. R., \& Ida, S. 2010, ApJ, 714, 1052

Showalter, M., Weaver, H., Buie, M., et al. 2013, in EGU General Assembly Conference Abstracts, Vol. 15, EGU General Assembly Conference Abstracts, 13786

Showalter, M. R., \& Hamilton, D. P. 2015, Nature, 522, 45

Showalter, M. R., Hamilton, D. P., Stern, S. A., et al. 2011, IAU Circ., 9221, 1

Showalter, M. R., Weaver, H. A., Stern, S. A., et al. 2012, IAU Circ., 9253, 1

Shu, F. H., \& Stewart, G. R. 1985, Icarus, 62, 360

Spaute, D., Weidenschilling, S. J., Davis, D. R., \& Marzari, F. 1991, Icarus, 92, 147

Stern, S. A. 1992, ARA\&A, 30, 185

—. 2008, Space Sci. Rev., 140, 3

Stern, S. A., Weaver, H. A., Steffl, A. J., et al. 2006, Nature, 439, 946

Stewart, G. R., \& Ida, S. 2000, Icarus, 143, 28

Süli, Á., \& Zsigmond, Z. 2009, MNRAS, 398, 2199

Supulver, K. D., Bridges, F. G., \& Lin, D. N. C. 1995, Icarus, 113, 188

Tholen, D. J., Buie, M. W., \& Grundy, W. M. 2012, LPI Contributions, 1667, 6327

Tholen, D. J., Buie, M. W., Grundy, W. M., \& Elliott, G. T. 2008, AJ, 135, 777

Toomre, A. 1964, ApJ, 139, 1217

Walsh, K. J., \& Levison, H. F. 2015, ArXiv e-prints, arXiv:1505.01208

Ward, W. R. 1997, Icarus, 126, 261

Ward, W. R., \& Canup, R. M. 2006, Science, 313, 1107

-. 2010, AJ, 140, 1168 
Weaver, H. A., Stern, S. A., Mutchler, M. J., et al. 2006, Nature, 439, 943

Weidenschilling, S. J. 1989, Icarus, 80, 179

Weidenschilling, S. J., Spaute, D., Davis, D. R., Marzari, F., \& Ohtsuki, K. 1997, Icarus, 128,429

Welsh, W. F., Orosz, J. A., Carter, J. A., et al. 2012, Nature, 481, 475

Wetherill, G. W., \& Cox, L. P. 1985, Icarus, 63, 290

Wetherill, G. W., \& Stewart, G. R. 1989, Icarus, 77, 330

—. 1993, Icarus, 106, 190

Winter, S. M. G., Winter, O. C., Guimarães, A. H. F., \& Silva, M. R. 2010, MNRAS, 404, 442

Wisdom, J. 1980, AJ, 85, 1122

Wyatt, M. C., Dermott, S. F., Telesco, C. M., et al. 1999, ApJ, 527, 918

Youdin, A. N., \& Kenyon, S. J. 2013, in Planets, Stars and Stellar Systems. Volume 3: Solar and Stellar Planetary Systems, ed. T. D. Oswalt, L. M. French, \& P. Kalas (Dordrecht: Springer Science \& Business Media), 1

Youdin, A. N., Kratter, K. M., \& Kenyon, S. J. 2012, ApJ, 755, 17

Young, E. F., \& Binzel, R. P. 1994, Icarus, 108, 219

Young, E. F., Young, L. A., \& Buie, M. 2007, in Bulletin of the American Astronomical Society, Vol. 39, AAS/Division for Planetary Sciences Meeting Abstracts \#39, 541 


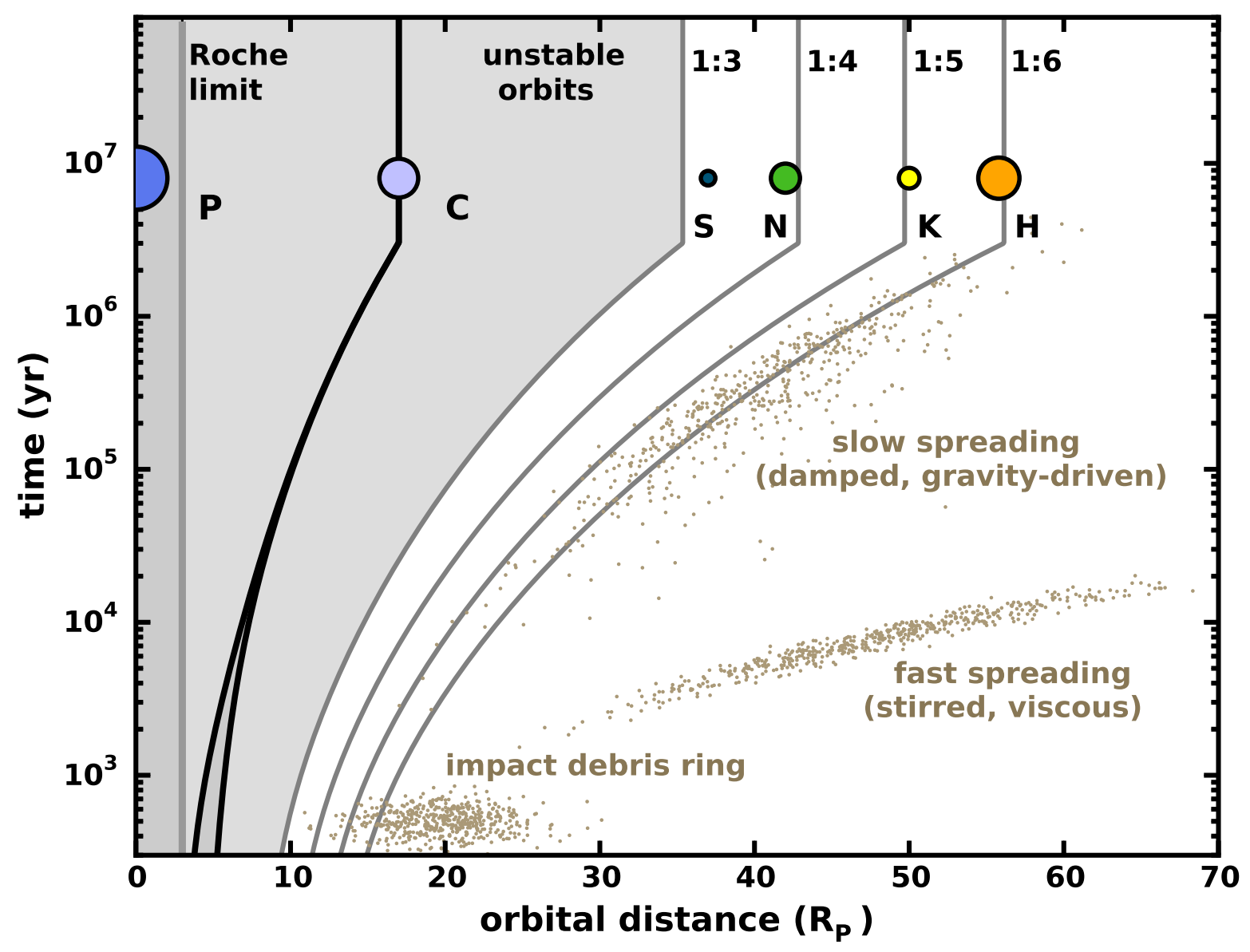

Fig. 1.- A schematic illustrating the layout and evolution of the Pluto-Charon satellite system. The binary is on the left. The satellites appear at their orbital distance from the center of mass, with symbol sizes scaled according to their approximate radii. The region inside of $3 R_{\mathrm{P}}$ is the Roche zone. Within this region, particles are held together by their own material strength; collisions dominate the particle dynamics. The light gray shaded region designates the inner cavity, where most planar orbits are unstable. The gray curves extending to large orbital distances locate commensurabilities (as labeled) as the binary expands. The brown dots at the lower left indicate the ring of debris after the binary formed, while the other clusters of brown dots refer to fast and slow modes of ring spreading. 


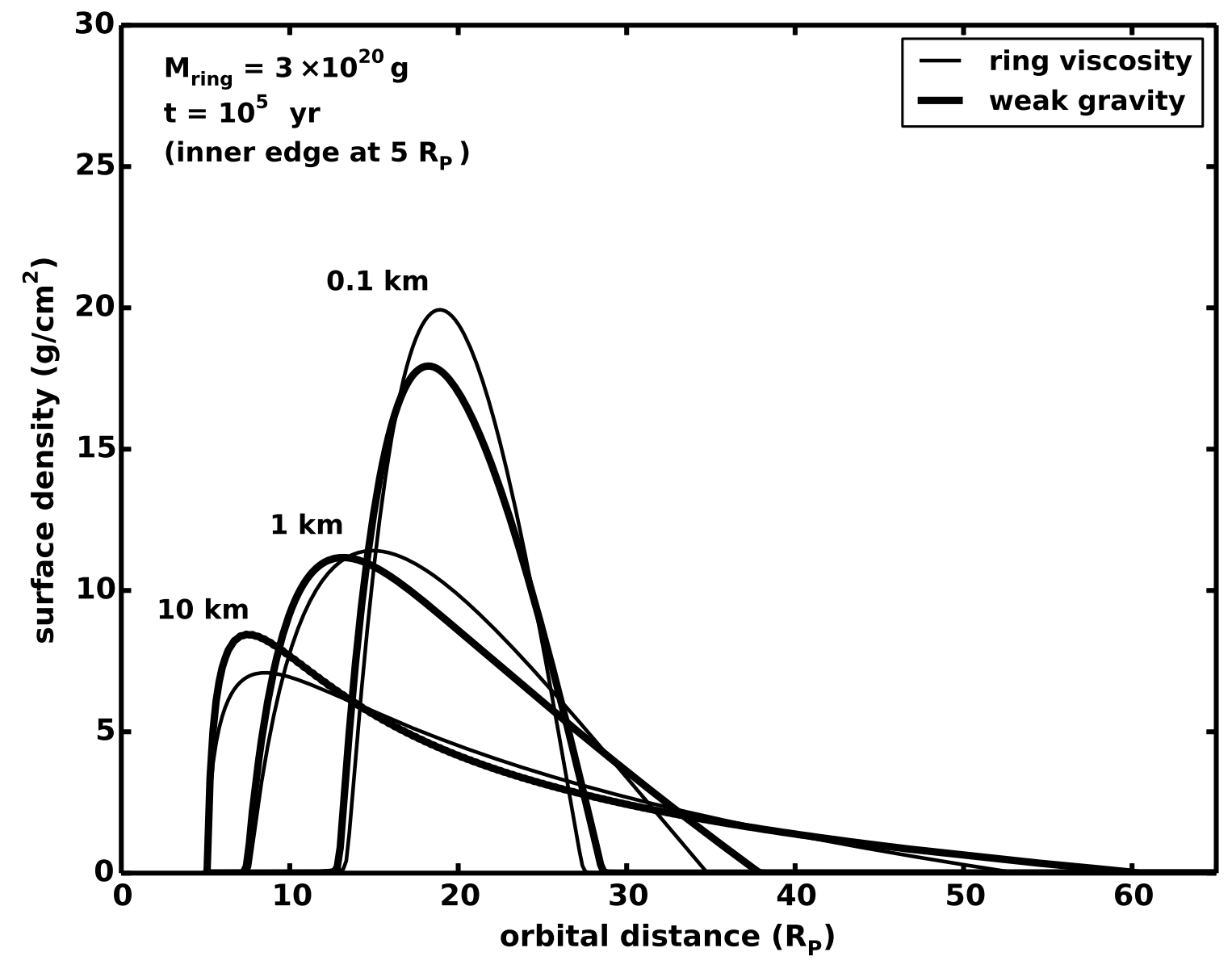

Fig. 2.- A comparison between the effects of viscosity and gravitational scattering in the spreading of rings. The astrophysical context is Pluto-Charon, at some early time when the binary is compact (orbital separation $a_{\mathrm{PC}}=5 R_{\mathrm{P}}$ ) and surrounded by a $3 \times 10^{20} \mathrm{~g}$ ring. Each scenario begins with the same surface density profile in the ring, but differ in the size of the ring particles, as indicated. In each case, the particles are stirred to their escape velocity. The dark curves spread from gravity only; light curves spread solely from viscosity. At this choice of particle speed, the two mechanisms should be similar. At higher speeds viscosity will dominate; at lower speeds gravity will drive the spreading. 

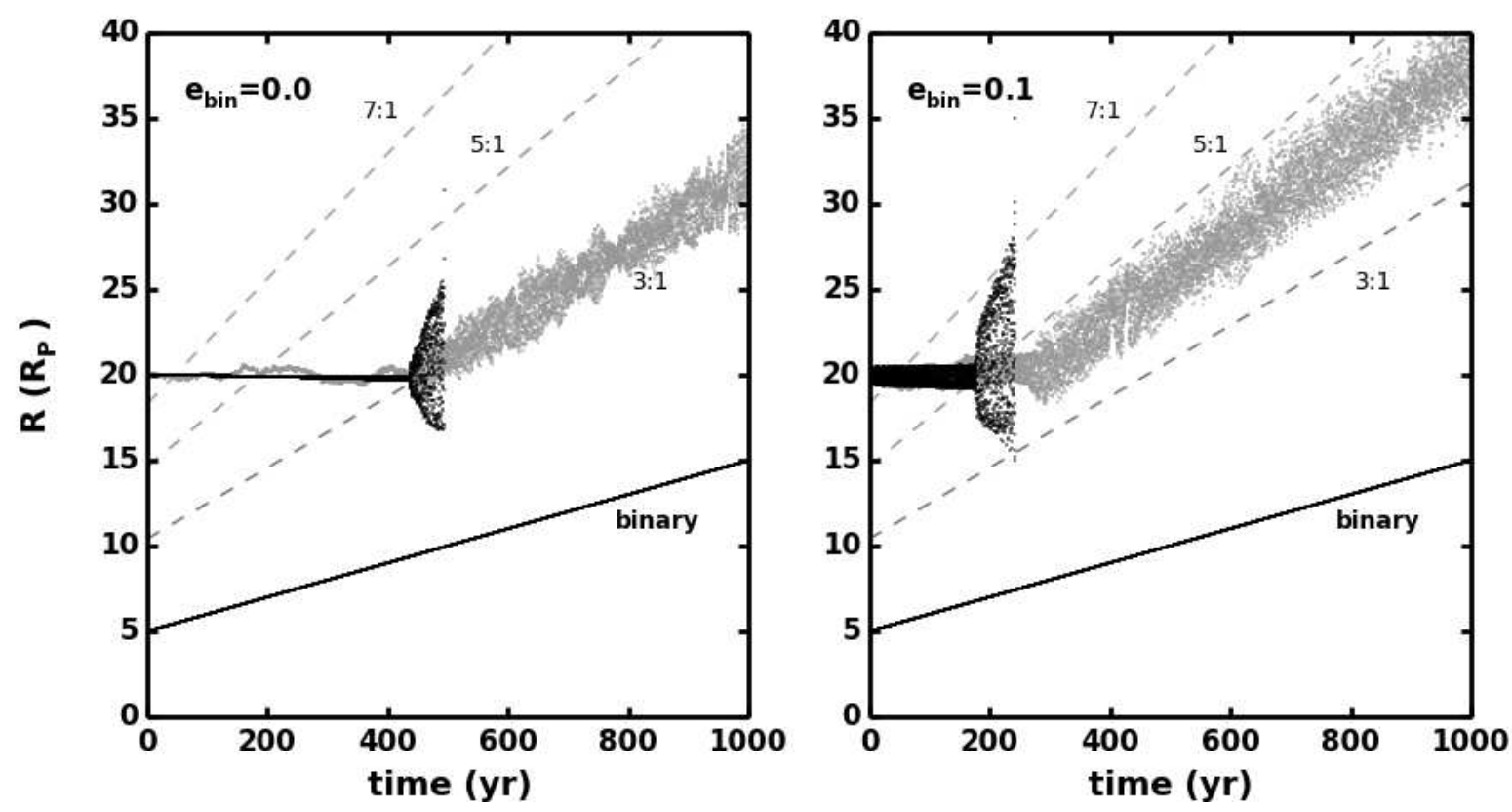

Fig. 3. - The orbital evolution of a satellite around an expanding binary. The left panel shows a circular "Pluto-Charon" binary expanding from an orbital separation of $5 R_{\mathrm{P}}$ (lower black curve). The dashed lines indicate the location of the 3:1, 5:1, and 7:1 commensurabilities expanding with the binary. A satellite on its own (black dots on the far left) orbits at a radial distance of $20 R_{\mathrm{P}}$ until it is overtaken by an instability near the 3:1 commensurability at about $400 \mathrm{yr}$. Then it is ejected from the system over a period of hundreds of orbits. A satellite that experiences collisional damping and radial diffusion (gray dots) survives the instability and "surfs" the 3:1 commensurability as it expands. The damping and diffusion parameters are drawn from fiducial values in $\$ 5.2\left(r=1 \mathrm{~km}, v=v_{\text {esc }}, \Sigma=40 \mathrm{~g} / \mathrm{cm}^{2}\right)$. The right panel is similar, but with an eccentric binary $\left(e_{\text {bin }}=0.1\right)$. In this case, an isolated satellite (black dots) is unable to navigate the 5:1 commensurability. A damped, diffusing satellite survives and rides resonances near the 4:1 commensurability. 


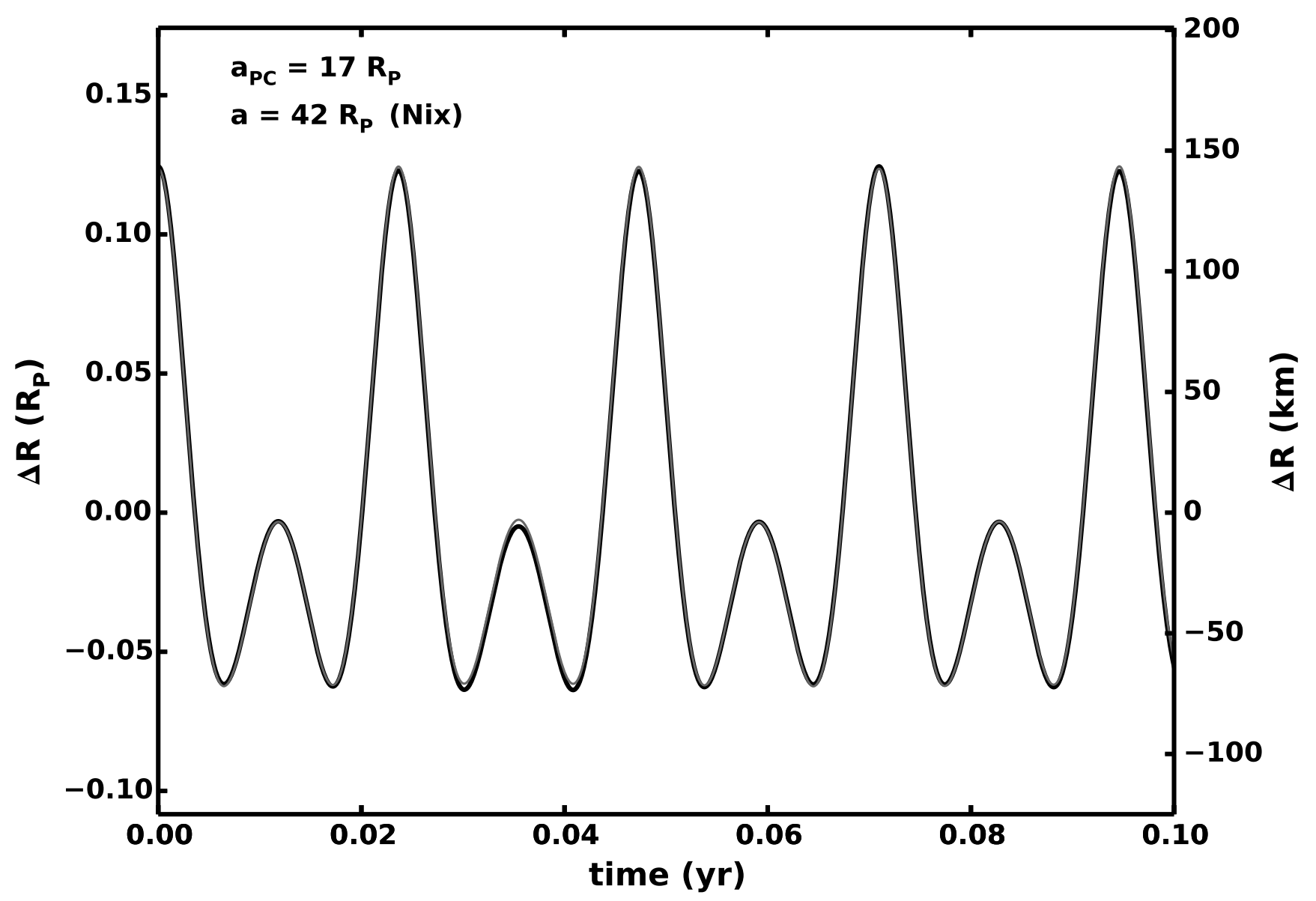

Fig. 4.- The radial excursion of a ring particle on a most circular circumbinary orbit for a binary with $e_{\text {bin }}=0$. The darker curve is from points calculated with a simulation starting from a best-fit to a minimum excursion orbit, while the lighter curve is from analytical theory (the two curves follow one another very closely). The binary separation and orbital distance correspond to the satellite Nix around Pluto-Charon. 


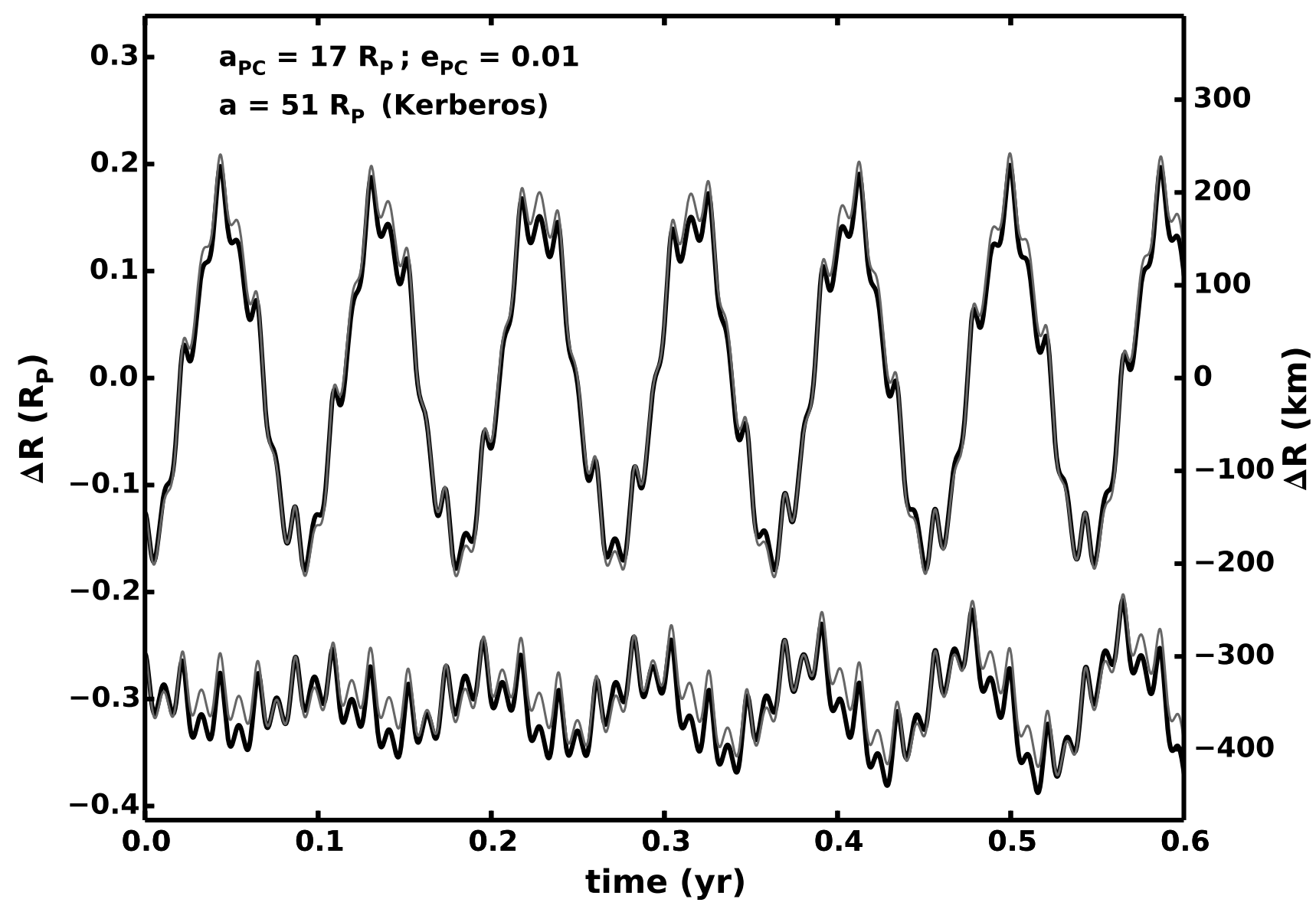

Fig. 5. - The radial excursion of ring particles in orbit about a binary with $e_{\text {bin }}=0.01$. The upper dark curve is for a most circular path, while the lower dark curve (displaced vertically for clarity) is for a particle that has equal measures of free and forced eccentricity. In time, the growth of the lower curve will continue; it will produce a beat pattern with a maximum amplitude equal to twice that of the most circular curve (e.g., Fig. 3 of Bromley \& Kenyon 2015). The light shaded curves are from analytical theory. The orbital elements are chosen to be similar to Kerberos around Pluto-Charon. 


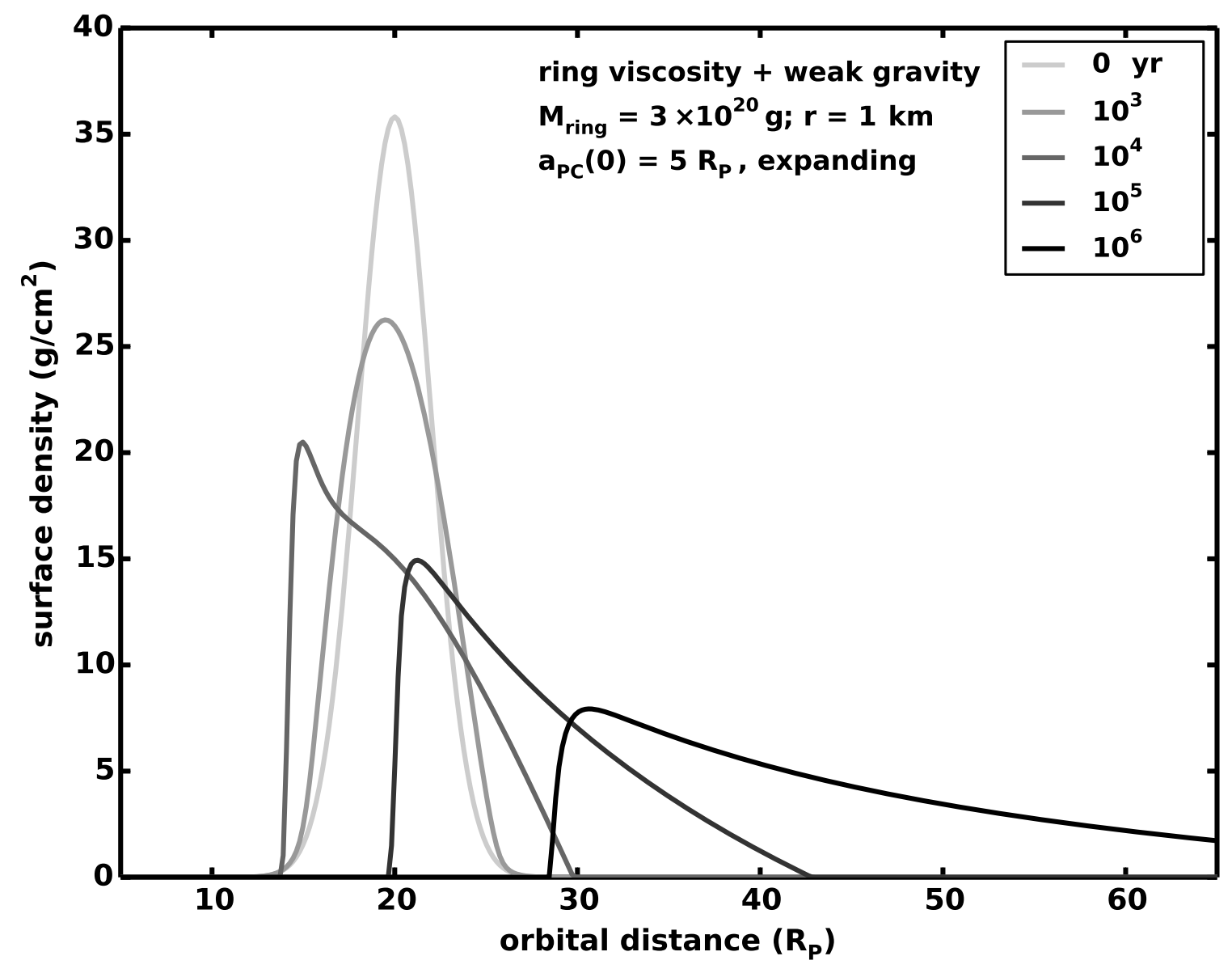

Fig. 6. - Spreading of a circumbinary ring. The curves show snapshots of the surface density as a function of orbital distance from Pluto-Charon. The rings spread from viscosity and gravitational scattering as the binary expands. The binary pushes the inner edge of ring outward, keeping it at a distance of twice the binary separation. 


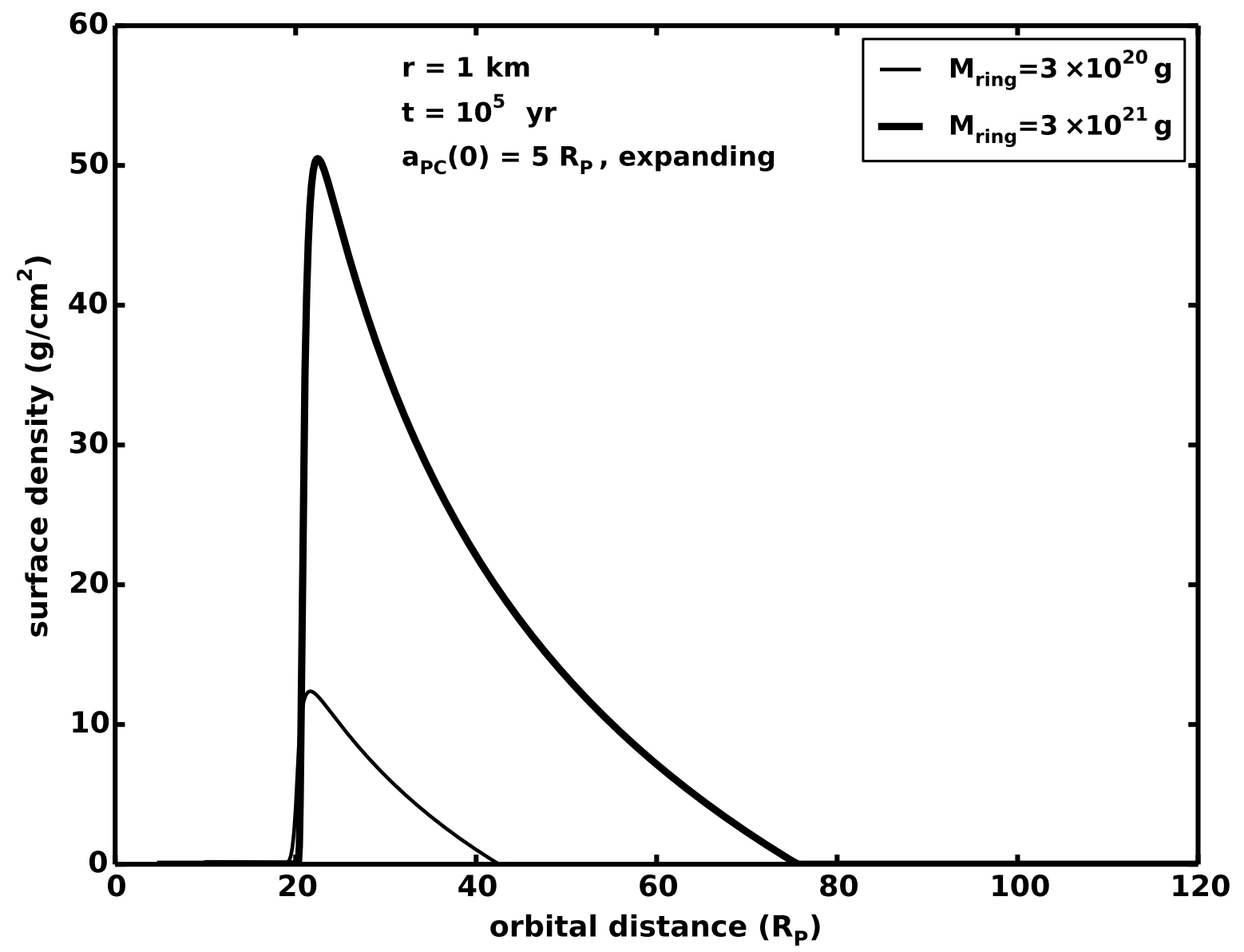

Fig. 7.- Spreading of circumbinary rings of two different masses as in the previous figure but with profiles shown only at $10^{5} \mathrm{yr}$. The rings initially differ only in their surface density; the outcomes show how the increased surface density drives the spreading. 


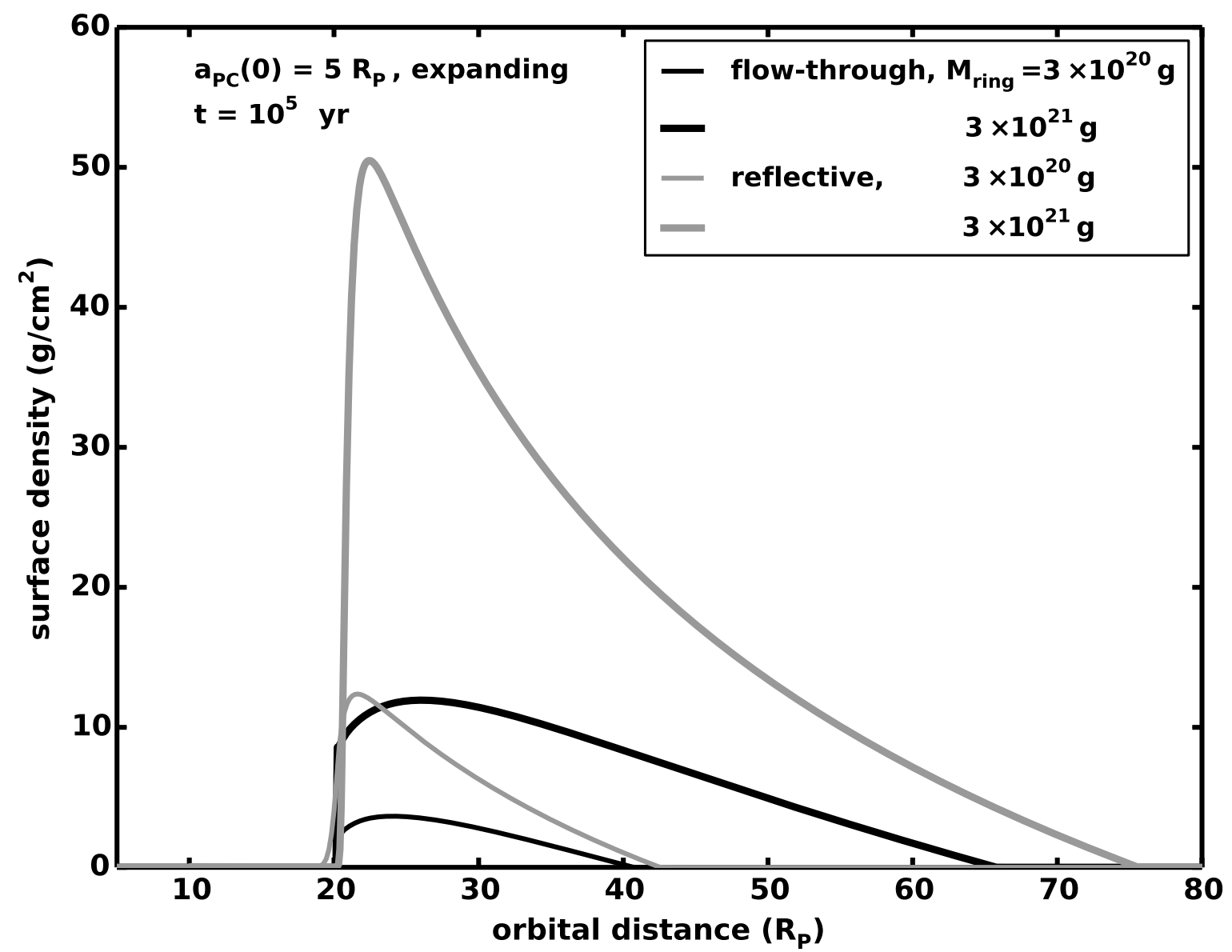

Fig. 8. - Spreading of a massive circumbinary ring with mass loss at the inner edge. The curves show the effect of mass loss at the inner edge of the ring, which expands along with the binary. 


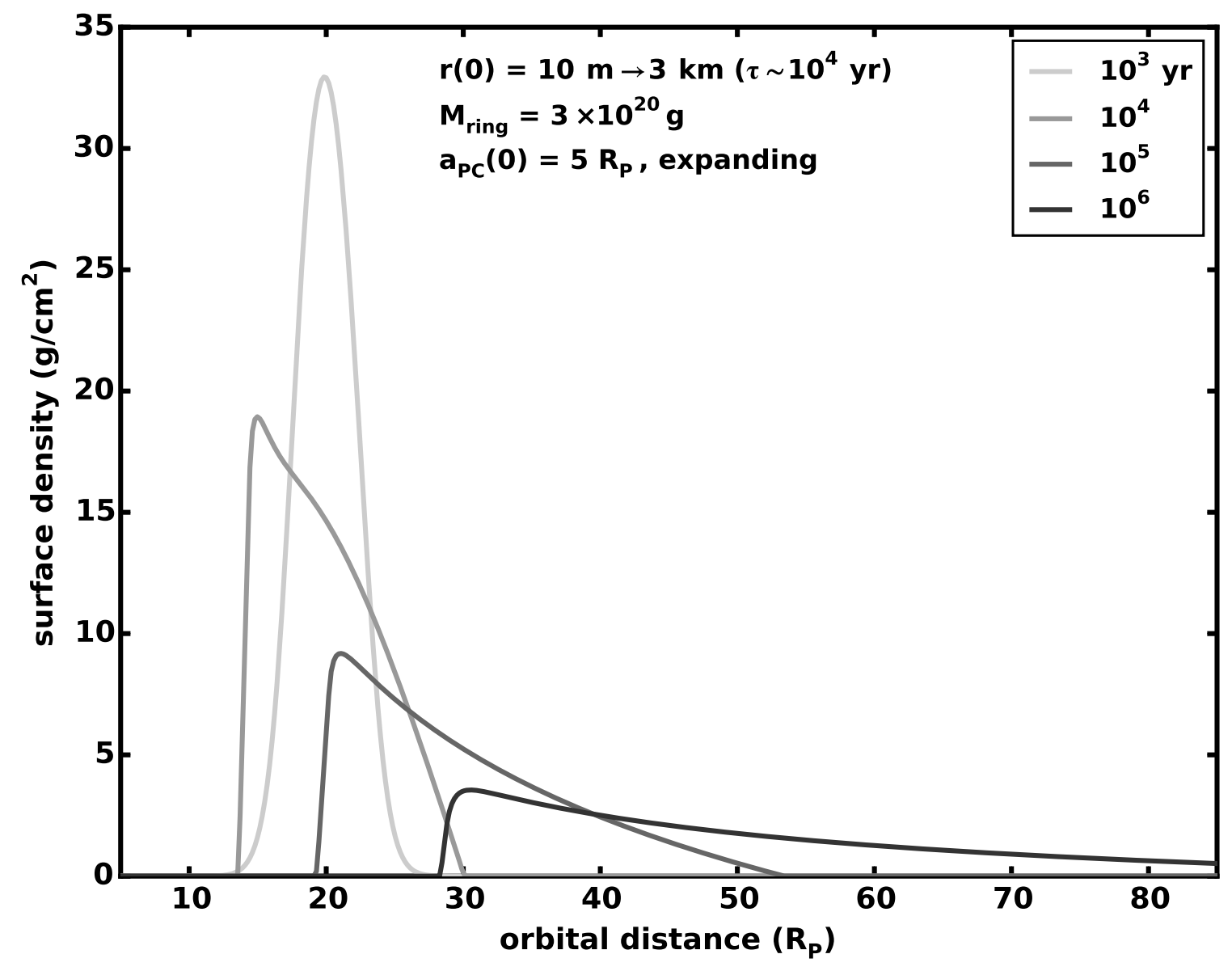

Fig. 9.- The spreading of a circumbinary ring with growing particles as in Figure 6 except the mass grows (along with the particle speeds, set by the escape velocity) from $10 \mathrm{~m}$ to $3 \mathrm{~km}$ on a time scale of $10^{4} \mathrm{yr}$. The growth allows for modest spreading beyond the baseline model with particle radii fixed at $1 \mathrm{~km}$. 


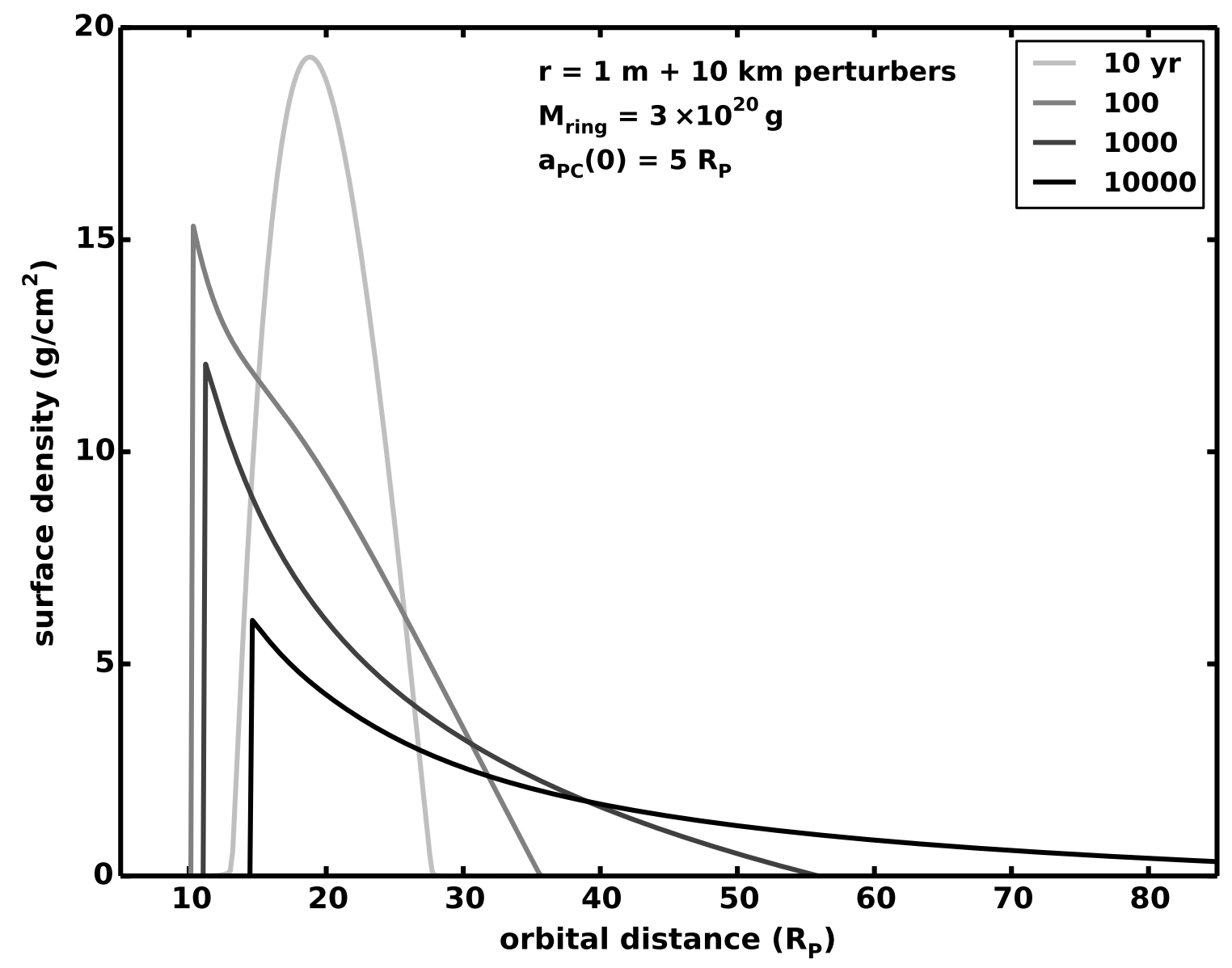

Fig. 10.- Rapid spreading of a circumbinary ring of small particles with embedded satellites. A population of meter-size objects stirred to 0.5 times the Hill velocity of $10-\mathrm{km}$ objects (evaluated at $20 R_{\mathrm{P}}$ ) spreads rapidly, as seen in the sequence of curves. In this case, material can flow through the inner edge of the ring. While this model is an idealization, it shows how effective viscous spreading can be when the relative velocities are pumped up. 